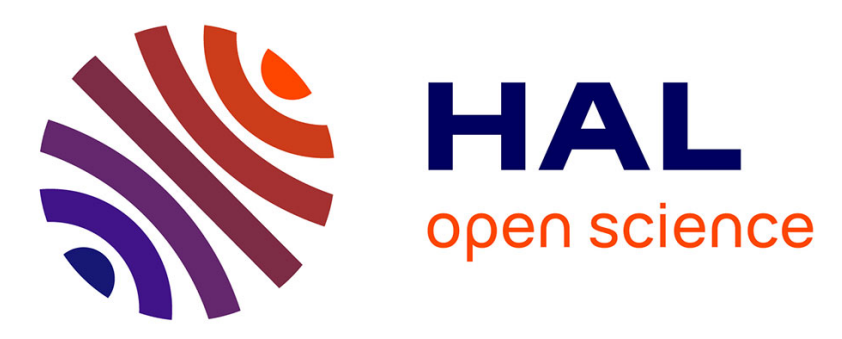

\title{
Finite element model of ionic nanowires with size-dependent mechanical properties determined by ab initio calculations
}

Julien Yvonnet, Alexander Mitrushchenkov, Gilberte Chambaud, Qi-Chang He

\section{- To cite this version:}

Julien Yvonnet, Alexander Mitrushchenkov, Gilberte Chambaud, Qi-Chang He. Finite element model of ionic nanowires with size-dependent mechanical properties determined by ab initio calculations. Computer Methods in Applied Mechanics and Engineering, 2011, 200 (5-8), pp.614-625. 10.1016/j.cma.2010.09.007 . hal-00725390

\section{HAL Id: hal-00725390 https://hal.science/hal-00725390}

Submitted on 10 May 2013

HAL is a multi-disciplinary open access archive for the deposit and dissemination of scientific research documents, whether they are published or not. The documents may come from teaching and research institutions in France or abroad, or from public or private research centers.
L'archive ouverte pluridisciplinaire HAL, est destinée au dépôt et à la diffusion de documents scientifiques de niveau recherche, publiés ou non, émanant des établissements d'enseignement et de recherche français ou étrangers, des laboratoires publics ou privés. 


\title{
Finite element model of ionic nanowires with size-dependent mechanical properties determined by ab initio calculations
}

\author{
J. Yvonnet*, A. Mitrushchenkov, G. Chambaud, Q.-C. He \\ ${ }^{a}$ Université Paris-Est, Laboratoire Modélisation et Simulation Multi Échelle, \\ MSME, UMR 8208 CNRS, 5 Bd Descartes, F-77454 Marne-la-Vallée Cedex 2, \\ France
}

\begin{abstract}
A finite element procedure for modeling crystalline nanostructures such as nanowires is proposed. The size effects exhibited by nano objects are captured by taking into account a surface energy, following the classical Gurtin Murdoch surface elasticity theory. An appropriate variational form and a finite element approach are provided to model and solve relevant problems numerically. We describe a simplified technique based on projection operators for constructing the surface elements. The methodology is completed with a computational procedure based on ab initio calculations to extract elastic coefficients of general anisotropic surfaces. The FEM continuum model is validated by comparisons with complete $a b$ initio models of nanowires with different diameters where size-dependent mechanical properties are observed. The FEM continuum model can then be used to model similar nanostructures in ranges of sizes or geometries where analytical or atomistic model are limited. The validated model is applied to the analysis of size effects in the bending of an AlN nanowire.
\end{abstract}

Key words: surface stress, size effects, nanowires, ab initio calculations

\section{Introduction}

Nanosized objects have at least one dimension in the nanometer range, roughly between 1 to $100 \mathrm{~nm}$. They are intermediate in size between atomistic/molecular and microscopic/continuum structures. By assembling nanostructural elements,

\footnotetext{
* Correspondance to J. Yvonnet

Email address: julien.yvonnet@univ-paris-est.fr (J. Yvonnet).
} 
it is possible to manufacture nanocomposites or nanodevices with desired properties and functions. Various types of nanostructural elements like nanowires, nanotubes, nanorods, nanorings, nanobelts or nanocombs have been synthesized and can serve as building blocks for nanosystems and nanostructures. In particular, 1D nanostructures such as nanowires have a great potential for use in nanoscale electronic components, field-effects transistors [2,10], ultra sensitive nanoscale gas sensors [11,69], nanoresonators [3], nanocantilevers [29], electromechanical oscillator [75], MEMS/NEMS [12,66,21] or microscopy probes [36]. For example, a nanogenerator which uses $\mathrm{ZnO}$ nanowires to convert mechanical energy to electrical energy has been successfully fabricated [61]. A review of mechanical effects in nanowires can be found in [45].

Nanowires and in general nanostructures are characterized by non zero surface energy that makes their effective properties size-dependent. Due to a different local environment, atoms near a free surface or interface have different equilibrium positions than do atoms in the bulk. Many experiments have reported such size-dependent behavior $[67,13,55,5,7,9,56,28,7,49]$. Due to the experimental difficulties related to very small sizes, measured properties for a given material are scattered and sometimes contradictory. An interesting related review and discussion can be found in [1].

The most straightforward methods to model nano objects are atomistic simulations either based on molecular dynamics (MD) or ab initio calculations. Studies employing MD to analyze size effects in nanostructures can be found for example in $[9,1,47,50,14]$. Works employing ab initio can be found e.g. in $[22,63,46,6]$. However, for objects characterized by a length larger than a few nanometers, the number of atoms in the systems becomes prohibitive to carry out computations. Multiscale numerical methods have been proposed in recent years (see e.g. $[58,23,48,60,42,70]$ among many others). Though very useful in many cases, these techniques inherently neglect some effects such as atomistic scale surface stress due to the fact that ghost, or pad atoms are necessary at the continuum/ atomistic boundary to ensure energy and force continuity [44]. An alternative is to construct continuum models augmented with surface energies related to the nanoscale effects.

Surface free energy is neglected in traditional continuum mechanics because it is associated with only a few layers of atoms. For objects with dimensions larger than dozen of nanometers, the ratio between volume and surface is extremely small. However, for nano-sizes particles, wires and films, this ratio becomes significant, and so does the effect of surface free energy. The importance of surface stress has led to important theoretical modeling advances that has started with the works of Gurtin and Murdoch [26]. In main approaches, nanosized bodies are generally considered to be made up of a "bulk" and a bounding "surface" in a continuum theory. In [35,15] a framework has been proposed to incorporate the surface energy into the continuum theory using a 
strain gradient approach, and used to model the size effects in nanobeams. To explain the size-dependence in their studies, Chen et al. [7] proposed a coreshell model, where the nanowire is treated as a composite with a shell and a core structure. This model assumes a Young modulus higher for the shell than for the bulk. In [15], Dingreville et al. proposed a methodology to model the size-dependent effective moduli of isolated nanosized elements (particles, wires and films). Effective properties of composites containing interface stress have been proposed in Duan et al. [18,19,17,62], Sharma et al. [52,53], and Le Quang and He [34], among others. For nanoparticles presenting sharp edges and corners, additional energetic terms related to line integrals can also be included [72]. This is especially important for 2D objects like graphene sheets [47]. It should be noted that the above-mentioned theoretical works are confined to systems of simple geometries, while systems with more complex geometries cannot be solved analytically. To further elucidate the surface elastic effect and characterize mechanical behaviour of nanosystems, numerical methods such as the finite element method (FEM) are promising alternatives. Wei et al. [64] and Tian et al. [59] proposed an FEM technique employing special surface elements to take into account surface elasticity. Yvonnet et al. [71] introduced an extended finite element (XFEM) methodology to include surface energy in nanosystems without any surface mesh requirement neither curvilinear coordinates. In a series of works, see e.g. [41-44], Park and coworkers developed an alternative continuum framework incorporating surface effects based on a the surface Cauchy-Born model. It extends the traditional Cauchy-Born theory $[4,57,65,58]$ by augmenting the continuum bulk energy density with a surface stress and takes into account nonlinear effects. In [32,33], Javili and Steinmann introduced formulations for solids with surface energies and related FEM discretization at finite strains. In [68] a different approach was used, considering a nanocantilever as an inhomogeneous bulk continuum materials whose local elastic constant are calculated from atomistic level.

In approaches in which surface properties are involved to model the size effects, a necessary step is the extraction of surface parameters. Shenoy and Miller [38,54] proposed a procedure based on MD calculations in the case of metallic crystals surfaces. Hamilton et al. [27] developed a similar technique to compute elastic coefficients of $\mathrm{Cu}(111)$ surfaces. In [22,63] slab models were employed to calculate surfaces coefficients via ab initio calculations. More recently, Zhang et al. [72], and Reddy et al. [47] have described methods to compute edges elastic properties in nanowires with sharp edges and in graphene sheets, respectively.

This work provides a complete procedure for modeling size-dependent mechanical effects in nanowires. Firstly, a continuum variational framework enriched with surface energy is developed, and a three-dimensional FEM methodology is presented to solve the problem numerically. The formulations are similar to the works of Javili and Steinmann $[32,33]$, though here restricted to stationnary 
and small strains. The main differences lie in the FEM discretization: in the present work the discrete surface operators are constructed without curvilinear coordinates, the surface projector operator components are directly embedded in the matrices. Secondly, a new technique based on ab initio computations is detailed to determine the surface parameters related to the continuum model. In an associated paper [39], we discuss in more details the physical phenomena at the atomistic scale and propose an analytical model of nanowire.

In section 2, we present the enriched continuum model equations and associated variational forms used within the FEM methodology, provided in section 3. In section 4 , the procedure to extract the elastic surface parameters based on $a b$ initio calculations is described. Validation tests and numerical examples are provided in section 6 . We first compare our continuum model with full AlN $a b$ initio nanowire models of different sizes. Then the validated model is used to analyze size effects in the bending analysis of a nanowire.

\section{Continuum modelling with surface energy}

We consider an open domain $\Omega \in \mathbb{R}^{3}$ with a bounding surface $\Gamma$. The latter $\Gamma$ is composed of two disjoint complementary parts $\Gamma_{u}$ and $\Gamma_{F}$ where the displacements and forces are prescribed, respectively. To introduce the surface energy and related size effects, the Gurtin-Murdoch model [26] of surface elasticity is adopted. The equilibrium equations of the problem are then given by

$$
\begin{aligned}
& \nabla \cdot \boldsymbol{\sigma}+\mathbf{b}=0 \text { in } \Omega, \\
& \nabla^{s} \cdot \boldsymbol{\sigma}^{s}+\boldsymbol{\sigma} \mathbf{n}=0 \text { on } \Gamma
\end{aligned}
$$

where $\boldsymbol{\sigma}$ is the Cauchy stress tensor, $\mathbf{b}$ is body force and $\mathbf{n}$ is the outward unit normal vector to $\Gamma$. Equation (1) refers to the bulk equilibrium equation, while Eq. (2) refers to the surface equilibrium (see e.g. [8]). Superscript (.) ${ }^{s}$ refers to surface quantities or operators. In Eq. (2), $\nabla^{s} \cdot \mathbf{T}$ denotes the surface divergence of a differentiable second-order tensor $\mathbf{T}$ and is defined by:

$$
\nabla^{s} \cdot \mathbf{T}=\nabla \mathbf{T}: \mathbf{P}
$$

In the above equation, the symbol ":" denotes double contraction of indices and $\mathbf{P}(\mathbf{x})=\mathbf{1}-\mathbf{n}(\mathbf{x}) \otimes \mathbf{n}(\mathbf{x})$ is an orthogonal projection operator describing the projection on the plane tangent to $\Gamma$ at $\mathbf{x} \in \Gamma$. The surface strain and stress tensors are defined by:

$$
\boldsymbol{\sigma}^{s}=\mathbf{P} \boldsymbol{\sigma} \mathbf{P}, \quad \varepsilon^{s}=\mathbf{P} \varepsilon \mathbf{P},
$$


where $\boldsymbol{\varepsilon}=\frac{1}{2}\left(\nabla \mathbf{u}+\nabla \mathbf{u}^{T}\right)$ is the linearized strain tensor and $\mathbf{u}$ is a displacement field. It is worth noting that the operator $\mathbf{P}$ does not involve any basis change: the reader has to keep in mind that $\mathbf{T}^{s}$ denotes the projection of $\mathbf{T}$ on the surface $\Gamma$. Thus $\mathbf{T}^{s}=\mathbf{P T P}$ does not express the components relative to the basis of the tangent plane to the surface. This projection tensor nicely allows one to mix bulk and surface quantities in one equation. The surface stress $\boldsymbol{\sigma}^{s}$ is related to the surface strain by

$$
\boldsymbol{\sigma}^{s}=\frac{\partial \gamma_{0}^{s}\left(\varepsilon^{s}\right)}{\partial \varepsilon^{s}}+\frac{\partial \gamma^{s}\left(\varepsilon^{s}\right)}{\partial \varepsilon^{s}}
$$

Above, $\gamma^{s}\left(\varepsilon_{0}^{s}\right)$ and $\gamma^{s}\left(\varepsilon^{s}\right)$ are specified by

$$
\gamma_{0}^{s}\left(\varepsilon^{s}\right)=\boldsymbol{\tau}^{s}: \varepsilon^{s}, \quad \gamma^{s}\left(\varepsilon^{s}\right)=\frac{1}{2} \varepsilon^{s}: \mathbb{C}^{s}: \boldsymbol{\varepsilon}^{s}
$$

Physically, $\gamma_{0}^{s}\left(\varepsilon^{s}\right)$ is an energy density due to surface residual stress $\boldsymbol{\tau}^{s}, \gamma^{s}\left(\varepsilon^{s}\right)$ is a surface strain density function with $\mathbb{C}^{s}$ denoting a surface elastic tensor. We do not assume an isotropic surface, i.e. $\boldsymbol{\tau}^{s} \neq \tau^{s} \mathbf{P}$. A discussion on the isotropic assumption of surfaces is proposed in [38]. The equations (1-2) are completed with boundary conditions prescribed on the surface $\Gamma_{u}$ and $\Gamma_{F}$ as

$$
\left\{\begin{array}{c}
\boldsymbol{\sigma} \mathbf{n}=\overline{\mathbf{F}} \text { on } \Gamma_{F} \\
\mathbf{u}(\mathbf{x})=\overline{\mathbf{u}}(\mathbf{x}) \text { on } \Gamma_{u} .
\end{array}\right.
$$

Finally, it is assumed that the surface adheres to the bulk:

$$
\llbracket \mathbf{u} \rrbracket=0 \text { on } \Gamma \text {. }
$$

It is worth noting that this model is a special case of a coherent imperfect interface [25] between two materials when one of them vanishes, thus the imperfect interface becomes a coherent membrane with its own elastic behavior.

To apply this framework to finite element analysis, we provide in the following the weak form of the equations. It can be derived straightforwardly by taking the classical weak form and taking into account a term related to the virtual internal work of the surface [71]. Another way to obtain it is to express the potential energy of the system and minimize the energy with respect to the displacement field. Thus, the potential energy of the system is given by:

$$
E=W^{b}(\varepsilon)+W^{s}\left(\varepsilon^{s}\right)-W^{e x t} .
$$

In the above expression, $W^{b}(\varepsilon)$ is the bulk elastic strain energy given by

$$
W^{b}(\varepsilon)=\int_{\Omega} \frac{1}{2} \varepsilon: \mathbb{C}^{b u l k}: \varepsilon d \Omega .
$$


In $(9), W^{s}\left(\varepsilon^{s}\right)$ is the surface elastic energy provided by

$$
W^{s}\left(\varepsilon^{s}\right)=\int_{\Gamma}\left[\gamma_{0}^{s}\left(\varepsilon^{s}\right)+\gamma^{s}\left(\varepsilon^{s}\right)\right] d \Gamma
$$

Finally, the work of external forces is expressed by:

$$
W^{e x t}=\int_{\Gamma_{F}} \mathbf{u} \cdot \overline{\mathbf{F}} d \Gamma+\int_{\Omega} \mathbf{u} \cdot \mathbf{b} d \Omega
$$

The equilibrium is found by writing the stationarity condition:

$$
D_{\delta \mathbf{u}} E=0
$$

where $D_{\mathbf{v}} f$ is the Gâteaux derivative of the functional $f$ in the direction $\mathbf{v}$. It leads to the weak form suitable for finite element discretization:

Find $\mathbf{u} \in H^{1}(\Omega)$ and $\mathbf{u}=\overline{\mathbf{u}}$ on $\Gamma_{u}$ such as

$$
\begin{aligned}
& \int_{\Omega} \boldsymbol{\varepsilon}(\mathbf{u}): \mathbb{C}^{b u l k}: \boldsymbol{\varepsilon}(\delta \mathbf{u}) d \Omega+\int_{\Gamma} \boldsymbol{\varepsilon}^{s}(\mathbf{u}): \mathbb{C}^{s}: \boldsymbol{\varepsilon}^{s}(\delta \mathbf{u}) d \Gamma \\
& =\int_{\Gamma_{F}} \overline{\mathbf{F}} \cdot \delta \mathbf{u} d \Gamma+\int_{\Omega} \mathbf{b} \cdot \delta \mathbf{u} d \Gamma-\int_{\Gamma} \boldsymbol{\tau}^{s}: \delta \boldsymbol{\varepsilon}^{s}(\delta \mathbf{u}) d \Gamma
\end{aligned}
$$

for all $\delta \mathbf{u} \in H_{0}^{1}(\Omega), H^{1}(\Omega)$ and $H_{0}^{1}(\Omega)$ being the usual Sobolev spaces.

\section{Finite element discretization}

In the following the Voigt notation is adopted. The vectorial form of the stress tensor is given by $[\boldsymbol{\sigma}]=\left\{\sigma_{11}, \sigma_{22}, \sigma_{33}, \sigma_{23}, \sigma_{13}, \sigma_{12}\right\}=\left\{\sigma_{1}, \sigma_{2}, \sigma_{3}, \sigma_{4}, \sigma_{5}, \sigma_{6}\right\}$ and the strain tensor counterpart is given by $[\varepsilon]=\left\{\varepsilon_{11}, \varepsilon_{22}, \varepsilon_{33}, 2 \varepsilon_{23}, 2 \varepsilon_{13}, 2 \varepsilon_{12}\right\}=$ $\left\{\varepsilon_{1}, \varepsilon_{2}, \varepsilon_{3}, \varepsilon_{4}, \varepsilon_{5}, \varepsilon_{6}\right\}$. The indices 1,2 and 3 correspond to the $\mathbf{x}, \mathbf{y}$ and $\mathbf{z}$ directions, respectively (see figure 1). In this work we consider the nanowire which is a monocrystal with wurtzite structure, as for example AlN, which grows with hexagonal cross-sections (see figure 3 ) and whose bulk elastic tensor can be expressed by 5 independent constants $C_{11}, C_{33}, C_{44}, C_{12}$ and $C_{13}$. The matrix form $\mathbf{C}^{\text {bulk }}$ of the bulk elastic tensor such that $[\boldsymbol{\sigma}]=\mathbf{C}^{b u l k}[\varepsilon]$ is given 


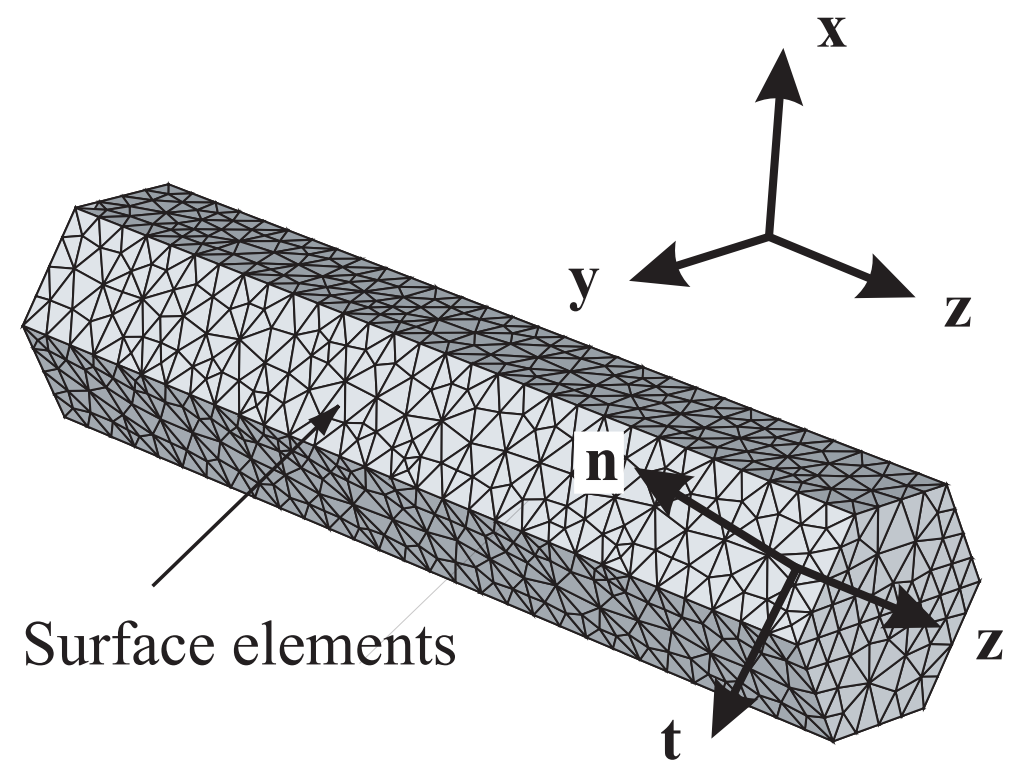

Fig. 1. Local basis and finite element model of nanowire with hexagonal cross section. by:

$$
\mathbf{C}^{b u l k}=\left[\begin{array}{cccccc}
C_{11}^{b} & C_{12}^{b} & C_{13}^{b} & 0 & 0 & 0 \\
C_{12}^{b} & C_{11}^{b} & C_{13}^{b} & 0 & 0 & 0 \\
C_{13}^{b} & C_{13}^{b} & C_{33}^{b} & 0 & 0 & 0 \\
0 & 0 & 0 & C_{44}^{b} & 0 & 0 \\
0 & 0 & 0 & 0 & C_{44}^{b} & 0 \\
0 & 0 & 0 & 0 & 0 & \frac{C_{11}^{b}-C_{12}^{b}}{2}
\end{array}\right]
$$

To solve the problem by FEM, it is necessary to discretize the weak form (14). While the bulk terms lead to classical matrix and vector forms, terms related to surface energy require a specific treatment. The domain is first discretized into volume elements, as shown in figure 1.

To discretize the surface terms, surface elements are required. In [71], we proposed a framework avoiding the explicit mesh of surfaces when considering surface energy by using an XFEM/level-set approach. In this work however, we adopt the classical FEM framework for the sake of simplicity. Let $\left(\mathbf{e}_{1}, \mathbf{e}_{2}\right)$ a local orthonormal basis related to the surface such that $\mathbf{e}_{1}=\mathbf{t}$ and $\mathbf{e}_{2}=\mathbf{z}$, with $\mathbf{z}$ and $\mathbf{t}=\mathbf{z} \times \mathbf{n}$ unit vectors along the main direction of the nanowire and tangent to the surface $\Gamma$ (see figure 1 ).

The surface residual stress can be expressed by:

$$
\boldsymbol{\tau}^{s}=\tau_{1}^{s} \mathbf{e}_{1} \otimes \mathbf{e}_{1}+\tau_{3}^{s} \mathbf{e}_{2} \otimes \mathbf{e}_{2}=\tau_{1}^{s} \mathbf{t} \otimes \mathbf{t}+\tau_{3}^{s} \mathbf{z} \otimes \mathbf{z}
$$


Due to the symmetry of the hexagonal nanowire, the six facets are identical and correspond to the $(10 \overline{1} 0)$ surfaces. Then the surface stress can be related to surface strain through four independent constants $C_{11}^{s}, C_{13}^{s}, C_{33}^{s}$ and $C_{55}^{s}$ and two residual stress components $\tau_{1}^{s}$ and $\tau_{3}^{s}$ :

$$
\left[\begin{array}{c}
\sigma_{1}^{s} \\
\sigma_{3}^{s} \\
\sigma_{5}^{s}
\end{array}\right]=\left[\begin{array}{ccc}
C_{11}^{s} & C_{13}^{s} & 0 \\
C_{13}^{s} & C_{33}^{s} & 0 \\
0 & 0 & C_{55}^{s}
\end{array}\right]\left[\begin{array}{c}
\varepsilon_{1}^{s} \\
\varepsilon_{3}^{s} \\
2 \varepsilon_{5}^{s}
\end{array}\right]+\left[\begin{array}{c}
\tau_{1}^{s} \\
\tau_{3}^{s} \\
0
\end{array}\right]
$$

The elastic tensor is expressed in the local basis as:

$$
\begin{aligned}
& \mathbb{C}^{s}=C_{11}^{s} \mathbf{t} \otimes \mathbf{t} \otimes \mathbf{t} \otimes \mathbf{t}+C_{33}^{s} \mathbf{z} \otimes \mathbf{z} \otimes \mathbf{z} \otimes \mathbf{z} \\
& +C_{13}^{s}(\mathbf{t} \otimes \mathbf{t} \otimes \mathbf{z} \otimes \mathbf{z}+\mathbf{z} \otimes \mathbf{z} \otimes \mathbf{t} \otimes \mathbf{t})+C_{55}^{s}(\mathbf{t} \otimes \mathbf{z} \otimes \mathbf{t} \otimes \mathbf{z}+\mathbf{z} \otimes \mathbf{t} \otimes \mathbf{z} \otimes \mathbf{t})(18)
\end{aligned}
$$

On substituting the FEM discretization into the weak form (14), and using the arbitrariness of nodal variations $\delta \mathbf{u}$, the following discrete system of linear equations is obtained:

$$
\left(\mathbf{K}+\mathbf{K}^{s}\right) \mathbf{q}=\mathbf{F}-\mathbf{F}^{s}
$$

with $\mathbf{q}$ being the vector of unknown displacements,

$$
\mathbf{K}=\int_{\Omega} \mathbf{B}^{T} \mathbf{C}^{b u l k} \mathbf{B} d \Omega
$$

being the bulk rigidity matrix and

$$
\mathbf{K}^{s}=\int_{\Gamma} \mathbf{B}^{T} \mathbf{M}_{p}^{T} \mathbf{C}^{s} \mathbf{M}_{p} \mathbf{B} d \Gamma
$$

being the surface rigidity matrix. In $(21) \mathbf{C}^{s}$ is the matrix form of the tensor (18) such as $\left[\boldsymbol{\sigma}^{s}\right]=\mathbf{C}^{s}\left[\varepsilon^{s}\right]$, with $\left[\boldsymbol{\sigma}^{s}\right]=\left\{\sigma_{11}^{s}, \sigma_{22}^{s}, \sigma_{33}^{s}, \sigma_{23}^{s}, \sigma_{13}^{s}, \sigma_{12}^{s}\right\}$ and $\left[\varepsilon^{s}\right]=$ $\left\{\varepsilon_{11}^{s}, \varepsilon_{22}^{s}, \varepsilon_{33}^{s}, 2 \varepsilon_{23}^{s}, 2 \varepsilon_{13}^{s}, 2 \varepsilon_{12}^{s}\right\}$. Note that $\left[\boldsymbol{\sigma}^{s}\right]$ is different from the left-hand term in (17) which express the surface stress in the local basis (tangent to the surface) while $\left[\boldsymbol{\sigma}^{s}\right]$ is expressed in the cartesian basis. As $\mathbf{C}^{s}$ is symmetric (see Eq. (18)), the matrix $\mathbf{K}^{s}$ is also symmetric. The matrix $\mathbf{M}_{p}$ relates the surface strains to the bulk strains through $\left[\varepsilon^{s}\right]=\mathbf{M}_{p}[\varepsilon]$. Its expression in $3 \mathrm{D}$ 
is specified by

$$
\begin{gathered}
\mathbf{M}_{p}=\left[\begin{array}{ccc}
P_{11}^{2} & P_{12}^{2} & P_{13}^{2} \\
P_{12}^{2} & P_{22}^{2} & P_{23}^{2} \\
P_{13}^{2} & P_{23}^{2} & P_{33}^{2} \\
2 P_{12} P_{13} & 2 P_{22} P_{23} & 2 P_{23} P_{33} \\
2 P_{11} P_{13} & 2 P_{12} P_{23} & 2 P_{13} P_{33} \\
2 P_{11} P_{12} & 2 P_{12} P_{22} & 2 P_{13} P_{23} \\
& \\
P_{12} P_{13} & P_{11} P_{13} \\
P_{22} P_{23} & P_{12} P_{23} & P_{11} P_{12} \\
P_{23} P_{33} & P_{13} P_{33} & P_{12} P_{22} \\
\left(P_{23}^{2}+P_{22} P_{33}\right) & \left(P_{23} P_{13}+P_{12} P_{33}\right) & \left(P_{22} P_{13}+P_{12} P_{23}\right) \\
\left(P_{13} P_{23}+P_{12} P_{33}\right) & \left(P_{13}^{2}+P_{11} P_{33}\right) & \left(P_{12} P_{13}+P_{11} P_{23}\right) \\
\left(P_{13} P_{22}+P_{12} P_{23}\right) & \left(P_{11} P_{23}+P_{13} P_{12}\right) & \left(P_{11} P_{22}+P_{12}^{2}\right)
\end{array}\right] .
\end{gathered}
$$

To compute (21), we define a set of surface elements $\mathcal{S}^{e}=\left\{\Gamma_{1}^{e}, \Gamma_{2}^{e}, \ldots, \Gamma_{M}^{e}\right\}$ such as $\bigcap_{i=1}^{M} \Gamma_{i}^{e}=\Gamma$. In the present case, $\Gamma_{i}^{e}$ are triangular facets. Let $\mathcal{S}^{\Gamma}$ be the set of tetrahedral elements having a triangular facet in $\mathcal{S}^{e}$ (see figure 2) and $\Omega^{e} \in \mathcal{S}^{\Gamma}$. We consider a bulk integration point $\mathbf{x}_{\Omega}^{e} \in \Omega^{e}$ and $\mathbf{x}_{\Gamma}^{e} \in \Gamma^{e}$ a surface integration point (see figure 2). If linear elements are considered, then the strain field is constant within each element $\Omega^{e}$. At the interface between elements the strain field is discontinuous. However, on the boundary we can extend the strain field to $\mathrm{x} \in \Gamma^{e}$ :

$$
\varepsilon\left(\mathbf{x}_{\Gamma}^{e}\right)=\lim _{\mathbf{x}_{\Omega}^{e} \rightarrow \mathbf{x}_{\Gamma}^{e}} \varepsilon\left(\mathbf{x}_{\Omega}^{e}\right)=\varepsilon\left(\mathbf{x}_{\Omega}^{e}\right)
$$

Then

$$
\left[\varepsilon^{s}\left(\mathbf{x}_{\Gamma}^{e}\right)\right]=\mathbf{B}\left(\mathbf{x}_{\Omega}^{e}\right)\left[\mathbf{u}^{e}\right]
$$

with $\left[\mathbf{u}^{e}\right]$ being the nodal displacement unknowns of $\Omega^{e}$.

We finally obtain the following approximation for $\mathbf{K}^{s}$ :

$$
\mathbf{K}^{s} \simeq \sum_{e \in \mathcal{S}^{\Gamma}} \mathbf{B}^{T}\left(\mathbf{x}_{\Omega}^{e}\right) \mathbf{M}_{p}^{T}\left(\mathbf{x}_{\Gamma}^{e}\right) \mathbf{C}^{s}\left(\mathbf{x}_{\Gamma}^{e}\right) \mathbf{M}_{p}\left(\mathbf{x}_{\Gamma}^{e}\right) \mathbf{B}\left(\mathbf{x}_{\Omega}^{e}\right)\left|\Gamma^{e}\right|
$$

where $\left|\Gamma^{e}\right|$ denotes the area of the triangular element $\Gamma^{e}$. Remark that integration points being located inside triangular facets, the unit normal vector 


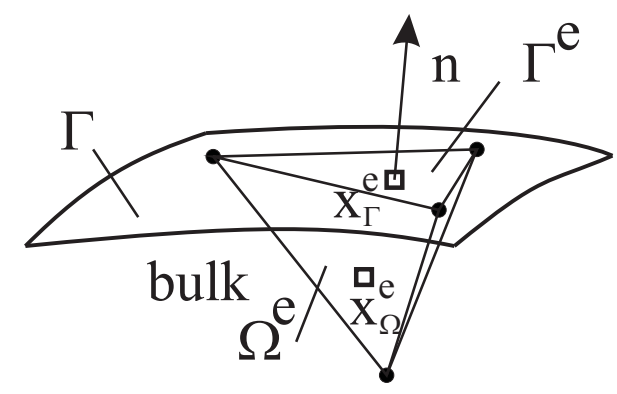

Fig. 2. integration points for surface elements.

$\mathbf{n}$ and tangent projector $\mathbf{P}$ are well defined. In this work we exclude the surface energies of both ends surfaces where displacement and external forces are prescribed. The generalized force vectors are obtained by

$$
\begin{aligned}
& \mathbf{F}=\int_{\Gamma_{F}} \mathbf{N}^{T} \overline{\mathbf{F}} d \Gamma+\int_{\Omega} \mathbf{N}^{T} \mathbf{b} d \Omega \\
& \mathbf{F}^{s}=\int_{\Gamma} \mathbf{B}^{T} \mathbf{M}_{p}^{T} \boldsymbol{\tau}^{s} d \Gamma
\end{aligned}
$$

where $\mathbf{N}$ is a classical FEM shape functions matrix. In the following, body forces are neglected.

\section{Extracting coefficients using Quantum Mechanics calculations}

\section{1 ab initio calculations}

In the present work, bulk and surface elastic parameters are extracted through atomistic $a b$ initio calculations. The above procedure has also been described in our associated paper [39]. A reason to employ ab initio calculations is to propose a framework that could be extended to piezoelectricity or coupled mechanical/chemical phenomena in future studies. Though methods such as core-shell models [74,37] can be used to introduce polarization within Molecular Dynamics, the validity of empirical potentials in the vicinity of defects and free surfaces is delicate. It is also true that ab initio calculations are limited for finite temperature calculations. However, as the present work is concerned with the study of surface effects, we want to remove possible artifacts related to empirical potentials that might lead to unphysical phenomena. Assuming 0 $\mathrm{K}$ temperature, elastic parameters are computed by deriving the energy with respect to the strains prescribed on a chosen unit cell. The total energy of the system if obtained as the lowest eigenvalue (ground state) of the electronic 
Schrödinger equation for fixed nuclei (Born-Oppenheimer approximation):

$$
\hat{H}_{e l}(\mathbf{r}, \mathbf{R}) \Psi_{k}(\mathbf{r}, \mathbf{R})=E(\mathbf{R}) \Psi_{e l}(\mathbf{r}, \mathbf{R})
$$

where $\hat{H}_{e l}$ is the electronic Hamiltonian operator, depending on the electrons positions $\mathbf{r}$, containing nuclear coordinates $\mathbf{R}$ as parameters. $\Psi_{k}$ is the electronic wave function and $E(\mathbf{R})$ is the total energy of the system as a function of nuclear coordinates. The explicit form of the Hamiltonian operator is

$$
\begin{aligned}
& \hat{H}_{e l}=-\frac{\hbar^{2}}{2 m_{e}} \sum_{i} \nabla_{i}^{2}+\sum_{\alpha} \sum_{\beta} \frac{Z_{\alpha} Z_{\beta} e^{2}}{d_{\alpha \beta}} \\
& -\sum_{\alpha} \sum_{i} \frac{Z_{\alpha} e^{\prime 2}}{d_{\alpha i}}+\sum_{j} \sum_{i>j} \frac{e^{\prime 2}}{d_{i j}} \\
& =(I)+(I I)+(I I I)+(I V)
\end{aligned}
$$

where the different terms are related to: (I) electrons kinetic energy, (II) nuclei $\alpha, \beta$ repulsion energy, (III) attraction between electrons and nuclei potential energy and (IV) electrons repulsion potential energy. For zero temperature we neglect nuclear vibrations. In above equations, $\hbar=h /(2 \pi), h$ being Planck's constant, $\alpha, \beta$ denote the indices related to nuclei, $m_{e}$ denotes electronic mass, $i, j$ denote the indices related to electrons and $\nabla_{i}^{2}$ are the Laplacian operators related to the $i$-th electron position. The parameter $Z_{\alpha}$ is the charge of nucleus $\alpha, e^{\prime}=e /\left(4 \pi \varepsilon_{0}\right)^{1 / 2}$ with $\varepsilon_{0}$ being the permittivity of vacuum and $d_{\alpha \beta}$ is the distance between two particles. Due to its high dimensionality, iterative and approximate methods are required to solve the Schrödinger equation such as Hartree-Fock (HF) or Density Functional Theory (DFT) methods [31].

In the present work we used the CRYSTAL code [16] for all ab initio calculations. B3LYP KS-DFT method with extra large space integration grid, was used. Basis set was $86-21 \mathrm{G}^{*}$ for $A l$ [40], and $6-31 \mathrm{Gd} 1 \mathrm{G}$ for $N[24]$. We have chosen the hybrid B3LYP functional because it is known to provide quite accurate energetic properties. As we are interested in elastic behavior, which is evaluated through energy derivatives, this choice seems appropriate.

\subsection{Bulk coefficients}

AlN nanowires have wurtzite structure related to the unit cell shown in figure 3 and described by the parameters $a=3.1111 \AA, c=4.9788 \AA$ and $u=0.3828$. 

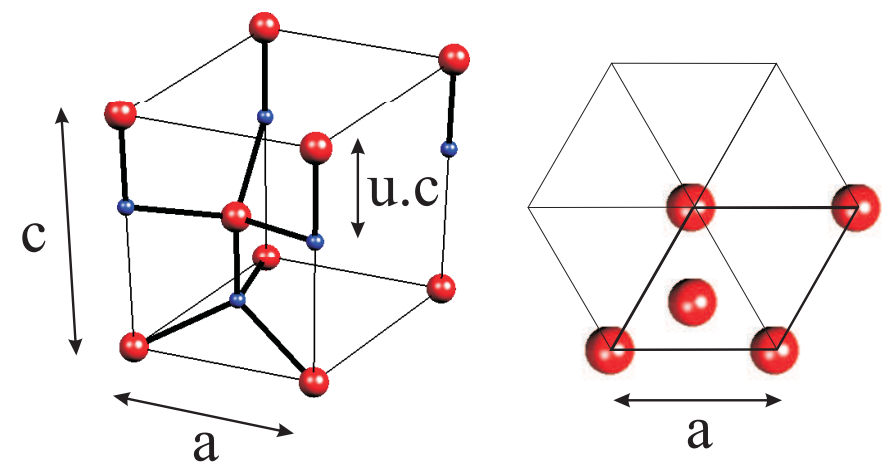

Fig. 3. Unit cell for wurtzite structure.

Table 1

Bulk elastic parameters (GPa).

\begin{tabular}{lcc}
\hline & Present work & Expt. \\
\hline$C_{11}^{b}$ & 421.0 & $410 \pm 10.0$ \\
$C_{33}^{b}$ & 405.4 & $388.5 \pm 10.0$ \\
$C_{12}^{b}$ & 133.2 & $148.5 \pm 10.0$ \\
$C_{13}^{b}$ & 100.1 & $98.9 \pm 10.0$ \\
$C_{44}^{b}$ & 129.9 & $124.6 \pm 4.5$ \\
\hline
\end{tabular}

Experimental values are provided from [30].

The unit cell volume $V$ is computed as

$$
V=a^{2} \frac{c \sqrt{2}}{2}
$$

The elastic constants are given by

$$
C_{i j}^{b}=\frac{1}{V} \frac{\partial^{2} E}{\partial \varepsilon_{i} \varepsilon_{j}} \quad i, j=1,2, \ldots, 6
$$

where $E=E_{0}$ is the total energy per unit cell (ground state energy) and $V$ is the unit cell volume. The total energy is determined using the DFT method described above for several values of strains $\varepsilon_{i}$ prescribed on the cell. When calculating elastic parameters, we fully relax internal atomistic coordinates for distorted unit cell. The data are fitted with a quartic polynomial which is derived to extract elastic constants. The obtained bulk elastic parameters are provided in Table 1 . These results agree well with previous works and experimental values [39]. 


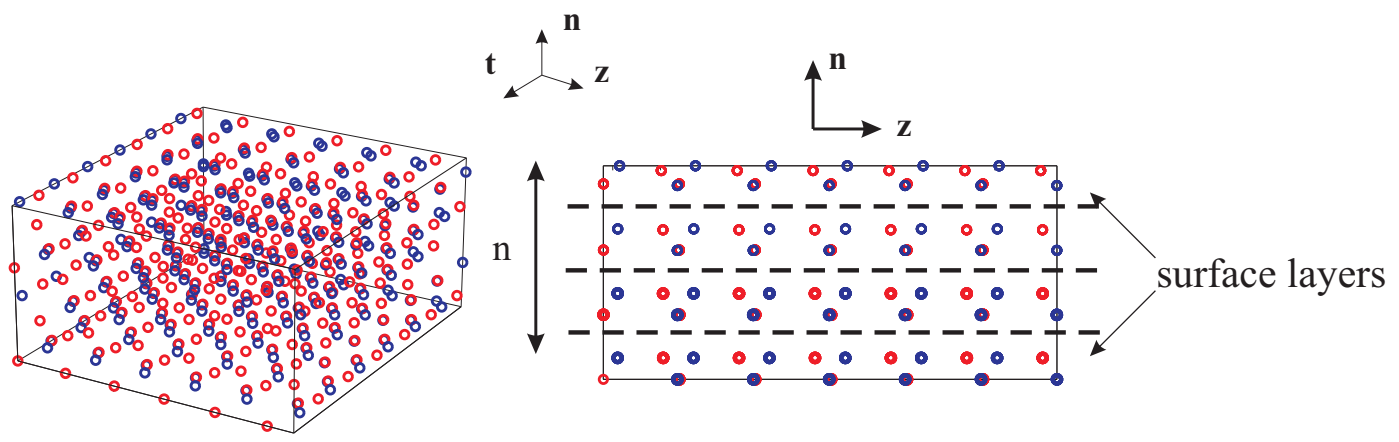

Fig. 4. Slab models with number of layers $n$.

\subsection{Surface coefficients}

To compute surface elastic parameters, a surface (slab) model shown in figure 4 is used, consisting of $n$ layers of atoms in the direction normal to the surface. Periodic conditions are applied along both other directions.

The possible strains for the slab system are $\varepsilon_{1}^{s}, \varepsilon_{3}^{s}$ and $\varepsilon_{5}^{s}$ which are prescribed along the $z$-(3) and $t$-(1) directions. The upper and lower surfaces normal to $\mathbf{n}$ are free to relax. The total energy of the system is the sum of two components, the surface energy and the bulk energy. The surface energy has to be isolated from the total energy. We assume the following model:

$$
E^{s l a b}(w)=w E^{s}+(1-w) E^{s l a b}(w \rightarrow 0)
$$

where

$$
w=\frac{2}{n}
$$

is the relative weight of the surface for a $n$-layer slab, as the model depicted in figure 4 contains two external atomic layers. Defining the elastic constants as derivatives of the energy with respect to strains, we obtain

$$
C_{i j}^{s l a b}(w)=w C_{i j}^{s}+(1-w) C_{i j}^{s l a b}(w \rightarrow 0)
$$

where $C_{i j}^{s}$ are the surface elastic properties and $C_{i j}^{s l a b}(w \rightarrow 0)$ are the limit values which can be obtained from bulk term by fully relaxing $\varepsilon_{2}$ for fixed $\varepsilon_{1}$ and $\varepsilon_{3}$ and optimizing the energy of the system. It yields [39]:

$$
\begin{aligned}
& C_{11}^{\text {slab }}(w \rightarrow 0)=\frac{\left(C_{11}^{b}\right)^{2}-\left(C_{12}^{b}\right)^{2}}{C_{11}^{b}} \\
& C_{13}^{\text {slab }}(w \rightarrow 0)=\frac{C_{13}^{b}\left(C_{11}^{b}-C_{12}^{b}\right)}{C_{11}^{b}}
\end{aligned}
$$




$$
\begin{aligned}
& C_{33}^{s l a b}(w \rightarrow 0)=\frac{C_{33}^{b} C_{11}^{b}-\left(C_{13}^{b}\right)^{2}}{C_{11}^{b}} \\
& C_{55}^{s l a b}(w \rightarrow 0)=C_{44}^{b} .
\end{aligned}
$$

Eq. (35) can be re-arranged as

$$
C_{i j}(w)=w\left(C_{i j}^{s}-C_{i j}^{s l a b}(w \rightarrow 0)\right)+C_{i j}^{s l a b}(w \rightarrow 0) .
$$

The procedure consists into computing values of $C_{i j}$ for different values of $w$ (by increasing the number of layers $n$ ) and fitting the obtained curve with a linear function to identify the slope $r=C_{i j}^{s}-C_{i j}^{s l a b}(w \rightarrow 0)$ and thus $C_{i j}^{s}$. For the residual stress we have:

$$
\tau_{i}(w)=w \tau_{i}^{s}
$$

In that case $\tau_{i}^{s}$ are directly obtained from the slope of the linear fit. We define per-area elastic constants as

$$
C_{i j}^{s l a b}=\frac{1}{S} \frac{\partial^{2} E^{s l a b}}{\partial \varepsilon_{i} \partial \varepsilon_{j}}
$$

where $S=a c$ is the unit cell area. The total surface energy $E^{s l a b}$ is determined using the DFT method described above for several values of strains $\varepsilon_{1}$, $\varepsilon_{2}$ and $\varepsilon_{5}$. As for bulk computations, a polynomial fitting was used to extract the derivatives. The resulting surface parameters are provided for the (10 $\overline{1} 0)$ AlN wurtzite surfaces in Table 2 (parameters A). In figure 5 the relative displacements in a slab model in free relaxation with $n=9$ layers is depicted, showing the surface effects. Al atoms are denoted by red circles while $\mathrm{N}$ atoms are denoted by blue circles.

\section{$5 \quad$ Remarks}

(1) In a previous work [71], a XFEM discretization was introduced to model the surface effects. In this paper, we have deliberately chosen not to introduce the XFEM for the sake of simplicity. In the case where a threedimensional mesh can be easily constructed for the domain and surface geometries, the XFEM procedure is not necessary. In that case, a standard FEM discretization procedure such as the one presented here is useful, as it allows employing classical FEM softwares. The cases studied in Yvonnet et al. [71], were more complex, involving microstructures with random distributions of voids, that justified the use of XFEM. Furthermore, it is worth noting that the present procedure introduced to 


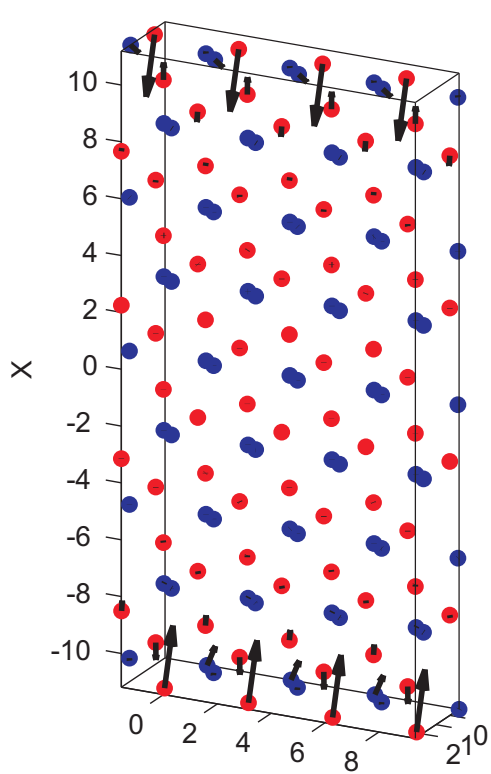

Z

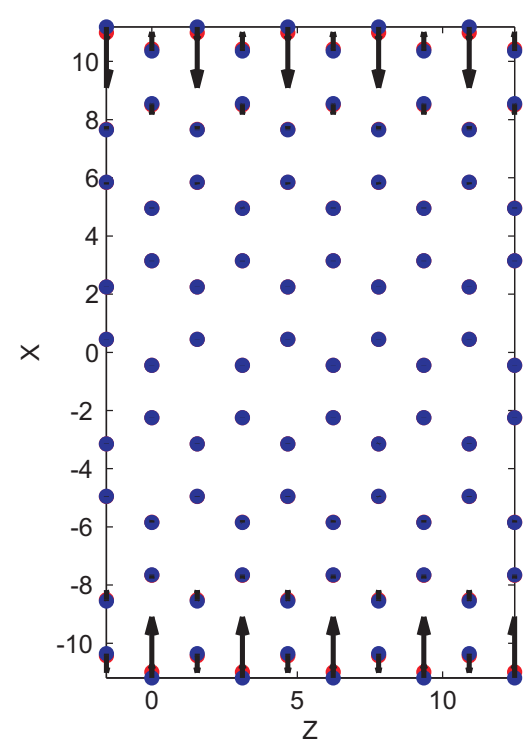

Fig. 5. Displacements of atoms in the slab model under free relaxation (red circles denote $\mathrm{Al}$ atoms, blue circles denote $\mathrm{N}$ atoms)

estimate the elastic coefficients via ab initio calculations is independent from the discretization technique.

(2) In Eq. (9), one alternative would be to integrate the bulk energy over a domain without the boundary layer occupied by a few atoms. However, it would lead to several difficulties: a) numerical integration would be more complicated in elements near the surface; b) the definition of the surface energy would then be delicate, as it would have to be weighted with respect to the nanowire diameter. A model with zero thickness has the advantage of avoiding these issues. However, the results obtained by directly using the coefficients from slab models are not always satisfactory and require adjusting the parameters. Quantifying error due to the difference between these two physical systems requires further theoretical and computational investigations which are beyond the scope of this first study.

\section{$6 \quad$ Numerical examples}

\subsection{Validation of the model: nanowire in traction}

The aim of this first example is to validate the mechanical continuum model by comparing it with ab initio calculations. Different atomistic models fully solved with an $a b$ initio method are constructed with increasing diameters. 


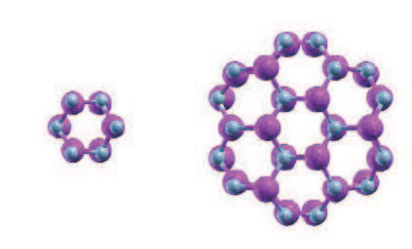

A

$$
\mathrm{n}=1 \quad \mathrm{n}=2
$$

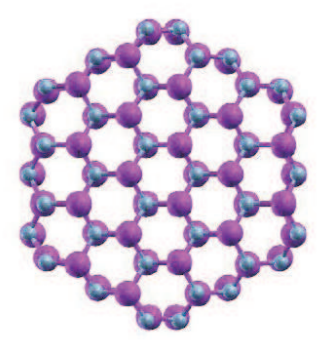

$\mathrm{C}$

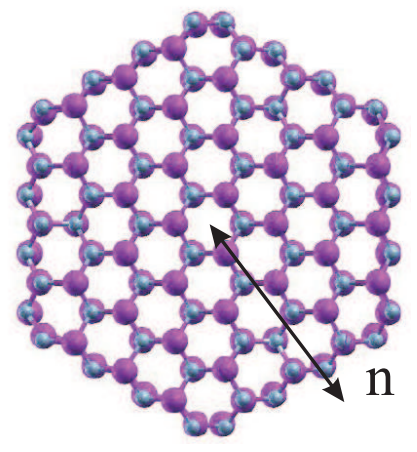

$\mathrm{D}$

Fig. 6. Atomistic models of nanowires from $n=1$ to $n=4$

We classify the nanowires according to the number of atomic layers $n$ in the nanowires (see figure 6). The biggest calculated nanowire corresponds to $n=7$ and contains 588 atoms in the unit cell. As both periodic conditions along z-axis and hexagonal symmetries are taken into account, a reduced model containing 98 atoms is employed. The cross sections corresponding to $n=1$ to $n=4$ are depicted in figure 6 .

We compute three quantities for both models: (a) the Young modulus $\bar{E}_{z z}$, (b) the axial residual strain $\bar{\varepsilon}_{z z}^{0}$, the axial strain of the nanowire in absence of loading due to surface residual stress $\boldsymbol{\tau}^{s}$ and (c) the bulk radial residual strain $\bar{\varepsilon}_{r r}^{0}$. This last value is defined as the radial strain in absence of loading taken at the center of a section. While the radial residual strain is not constant within the section, as seen in the following, it is quite constant in a region near the center of the section. This provides a comparable quantity for both models regarding the radial strain, as the diameter variation cannot be defined due to the complex deformation of the surface.

To compute the Young modulus of the ab initio nanowire model, a strain along the Z-direction of the nanowire is prescribed while the atoms in the other directions are free to relax. The energy is then computed by the $a b$ initio method described above for different strains and used to calculate

$$
\bar{E}_{z z}^{a b i n i t}=\frac{1}{V^{h}} \frac{\partial^{2} E}{\partial \varepsilon_{z}^{2}},
$$

$V^{h}$ being the volume of the hexagonal periodic layer. In the case of the FEM/continuum, we compute the Young's modulus by blocking the Z- degrees of freedom on one hexagonal facet and prescribing a stress distribution $\overline{\mathbf{F}}=\boldsymbol{\sigma} \mathbf{n}=\bar{\sigma}_{z z}\left(\mathbf{e}_{z} \otimes \mathbf{e}_{z}\right) \mathbf{n}$ on the other end. The problem being linear, the 
Young's modulus is evaluated as

$$
\bar{E}_{z z}^{F E M} \approx \frac{\bar{\sigma}_{z z}}{\bar{\varepsilon}_{z z}-\bar{\varepsilon}_{z z}^{0}}
$$

where $\bar{\varepsilon}_{z z}$ is the strain of the nanowire corresponding to $\bar{\sigma}_{z z}$. To reproduce the simulation conditions of ab initio calculations, where strain is applied to the system, we carried out another series of tests to estimate the effective Young modulus. For this purpose, we prescribed macroscopic strains along z-direction, while allowing relaxation in the radial direction, and computed the effective energy of the system for each one. The Young modulus was then obtained by the second-derivative of the effective energy, estimated by finite differences. An FEM model of a nanowire with hexagonal cross-section, diameter $d$ and length $L=4 d$ is meshed with tetrahedra as shown in figure 1. Different meshes with increasing element densities have been used to test the convergence of the solution, with 1344, 8905 and 24266 elements, and 361, 2035 and 4815 nodes, respectively.

In the present case, the bulk Young modulus along the $\mathrm{z}$ - direction can be calculated by first relaxing radial strains in the energy expression and then deriving twice this expression with respect to the axial (z) strain. Thus, we obtain:

$$
\bar{E}_{z z}^{b}=\frac{C_{33}^{b}\left(C_{11}^{b}+C_{12}^{b}\right)-2\left(C_{13}^{b}\right)^{2}}{C_{11}^{b}+C_{12}^{b}} .
$$

Using the numerical values of Table I obtained from our ab initio calculations we find $E_{z z}^{b}=369.239 \mathrm{GPa}$. Setting zero values to the surface parameters, we find numerically 369.23972 GPa using both methods for the mesh with 24266 elements.

To evaluate $\bar{\varepsilon}_{r r}^{0}$, an element containing the center of the section is selected. Then the strain tensor in the radial basis $\tilde{\boldsymbol{\varepsilon}}=\mathbf{R}^{T} \boldsymbol{\varepsilon} \mathbf{R}$ is computed, $\mathbf{R}$ being a rotation matrix related to the radial basis. For FEM curves, two results are provided, denoted by Continuum A and Continuum B. The model A employs the surface parameters obtained directly from the slab calculations, without any corrections. Model B uses parameters which are optimized so as to fit the three $\bar{E}_{z z}, \bar{\varepsilon}_{z z}^{0}$ and $\bar{\varepsilon}_{r r}^{0}$ ab initio curves. The initial parameters obtained from the slab procedure were taken to be initial guess for the optimization procedure. The obtained surface parameters are reported in Table 2.

Results are presented in figures 7, 8, 9 and 10. The mesh containing 8905 nodes was used to plot the continuous curves.

We can observe from figure 7 that the agreement between the continuum and $a b$ initio models is good in regards to Young's modulus, when employing the 
Table 2

Surface elastic parameters $(\mathrm{N} / \mathrm{m})$.

\begin{tabular}{ll}
\hline Parameters A (obtained from slab calculations) & Parameters B (optimized) \\
\hline$C_{33}^{s}=78.0$ & $C_{33}^{s}=93.59$ \\
$C_{11}^{s}=114.1$ & $C_{11}^{s}=114.1$ \\
$C_{13}^{s}=22.3$ & $C_{13}^{s}=22.3$ \\
$C_{55}^{s}=44.0$ & $C_{55}^{s}=44.0$ \\
$\tau_{1}^{s}=-2.33$ & $\tau_{1}^{s}=-3.33$ \\
$\tau_{3}^{s}=-0.873$ & $\tau_{3}^{s}=-1.533$ \\
\hline
\end{tabular}

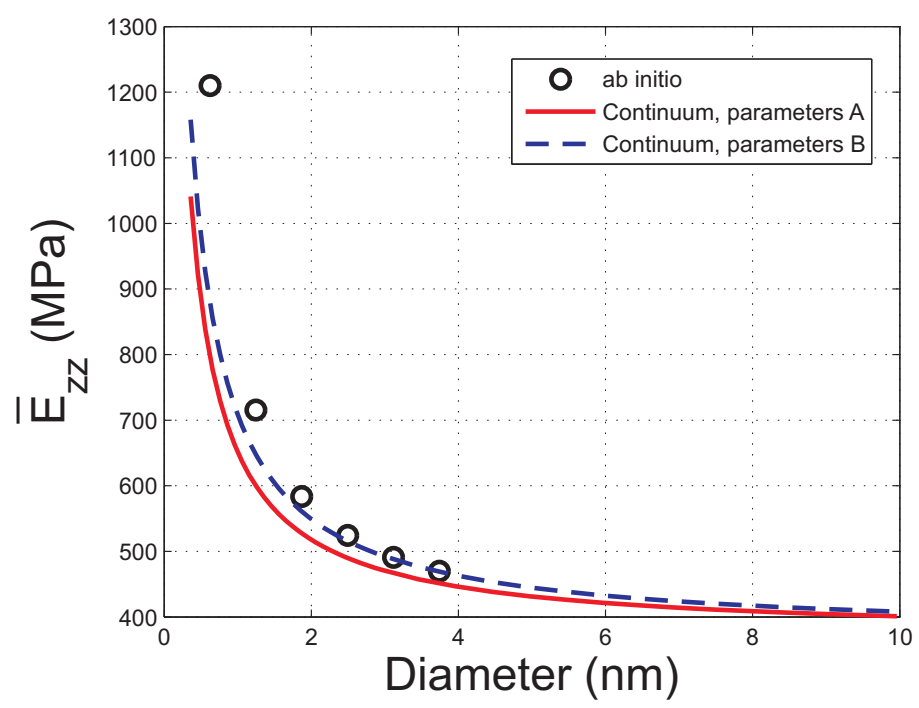

Fig. 7. Effective Young modulus of the nanowire: comparison between continuum FEM model and $a b$ initio model (prescribed stress).

coefficients obtained from the slab procedure without any modification. The use of the optimized coefficients gives rise to a slight improvement. Using the energy method, we obtain slightly better results than using the applied stress, as shown in figure 8 .

In figure 9 , we notice a significant discrepancy with respect to the ab intio solution when the continuum model is applied with the coefficients A for computing $\bar{\varepsilon}_{z z}$. Several sources of errors are possible: a) the slab surface assumes an infinite surface, while the surfaces are actually finite in the hexagonal nanowire; b) we did not take into account the energy of the edges corresponding to the intersection of the planes containing the facets of the nanowire. As reported in other works, these energy are in some cases not negligible for small nanoparticles sizes [73], c) additional couplings between surface and bulk due to the hexagonal symmetry might occur [39], which cannot be captured by the present slab model. However, using the optimized coefficients B, a very good 


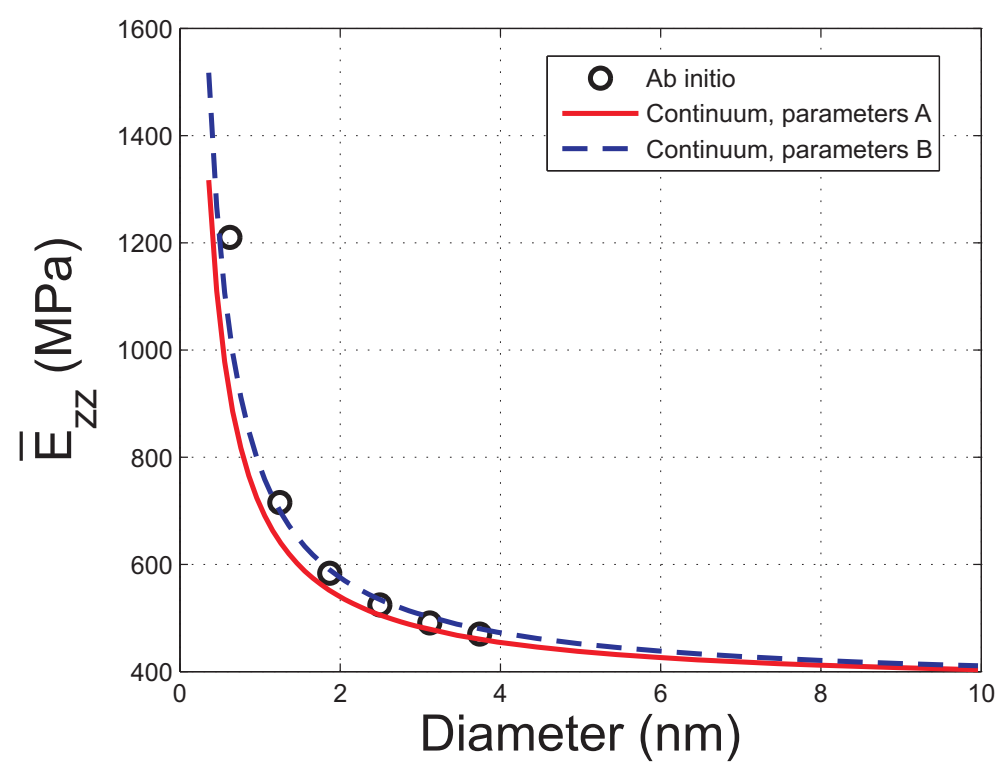

Fig. 8. Effective Young modulus of the nanowire: comparison between continuum FEM model and $a b$ initio model (prescribed strain).

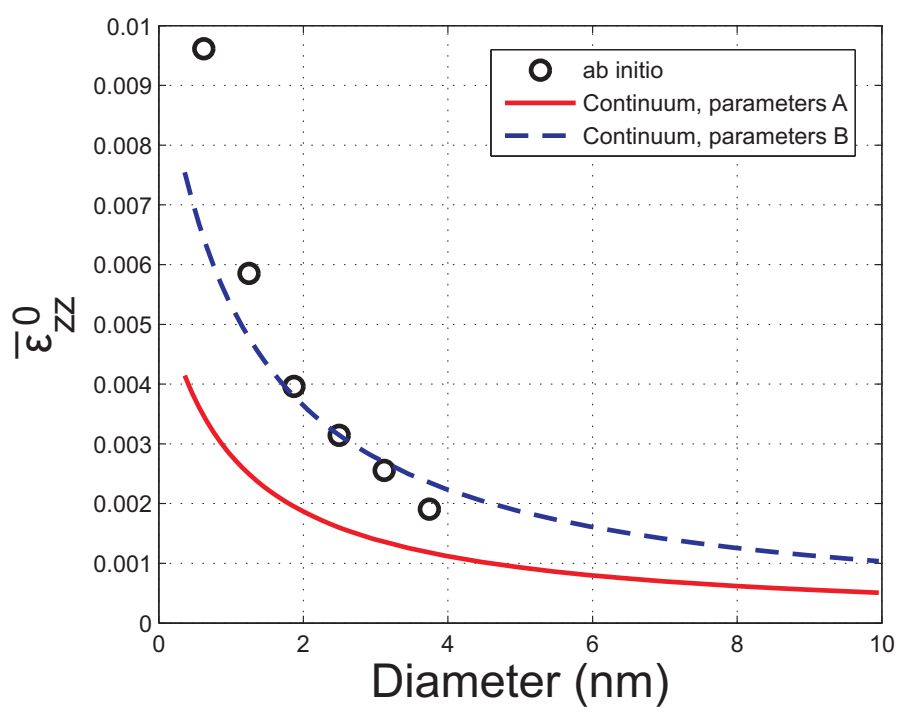

Fig. 9. Nanowire axial strain in relaxation: comparison between continuum FEM model and $a b$ initio model

agreement is observed.

In contrast, the agreement for $\bar{\varepsilon}_{r r}$ is satisfactory when one uses the slab parameters A. As expected, the optimized coefficients B lead to an even better accuracy of the model.

In table 3 we provide the values of $\bar{E}_{z z}, \bar{\varepsilon}_{z z}^{0}$ and $\bar{\varepsilon}_{r r}^{0}$ computed with the continuum FEM model for different mesh densities to test the convergence of the solution. A diameter $d=1 \mathrm{~nm}$ was chosen, and the surface parameters A were 


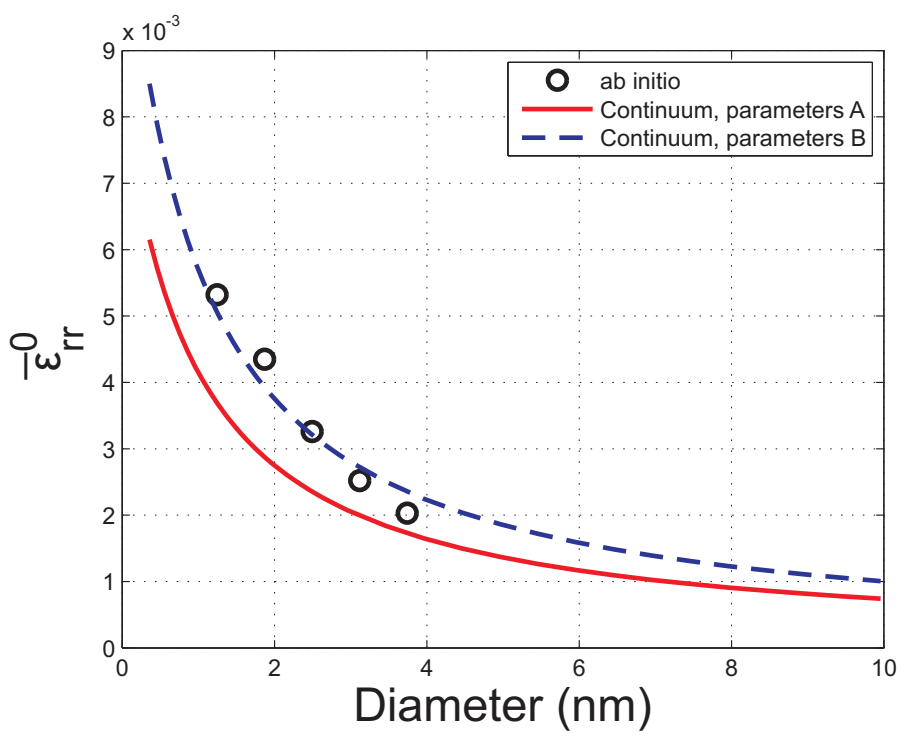

Fig. 10. Nnaowire Bulk radial strain in relaxation: comparison between continuum FEM model and $a b$ initio model

Table 3

Convergence of the computed values versus mesh size.

\begin{tabular}{llll} 
Nb. elements & 1344 & 8905 & 24266 \\
\hline $\bar{E}_{z z}(\mathrm{GPa})$ & 683.2 & 651.3 & 649.4 \\
$\bar{\varepsilon}_{z z}^{0}$ & 0.002788 & 0.00280699 & 0.00280172 \\
$\bar{\varepsilon}_{r r}^{0}$ & 0.005472 & 0.004150 & 0.0039808 \\
\hline
\end{tabular}

used.

From the above results, we conclude that the model has a good accuracy regarding the effective properties of the nanowire. In what follows, we examine the local fields. A continuous displacement field is constructed from the $a b$ initio discrete atomic displacements. For this purpose, a Delaunay mesh is constructed on the basis of the atomic coordinates. As the set of atomic centers does not form a convex hull, the spurious tetrahedra are removed using a maximum radius criterion (alpha-shape technique [20]). Then the displacement field is interpolated on the basis of linear FEM shape functions related to the tetrahedra. The absolute value of the continuous radial displacement solution is computed along the main radius of the nanowire and compared for both models in figures 11 and 12, corresponding to the nanowires with diameters $d=1.87 \mathrm{~nm}(\mathrm{n}=4)$ and $d=3.11 \mathrm{~nm}(\mathrm{n}=6)$, respectively.

We can notice from figures 11 and 12 that in the bulk region both displacement fields are in very good agreement, especially when the set of optimized surface coefficients B is used. However, near the surface, the local fields are poorly reproduced. This can be partly explained by the fact that both $\mathrm{Al}$ and $\mathrm{N}$ atoms 


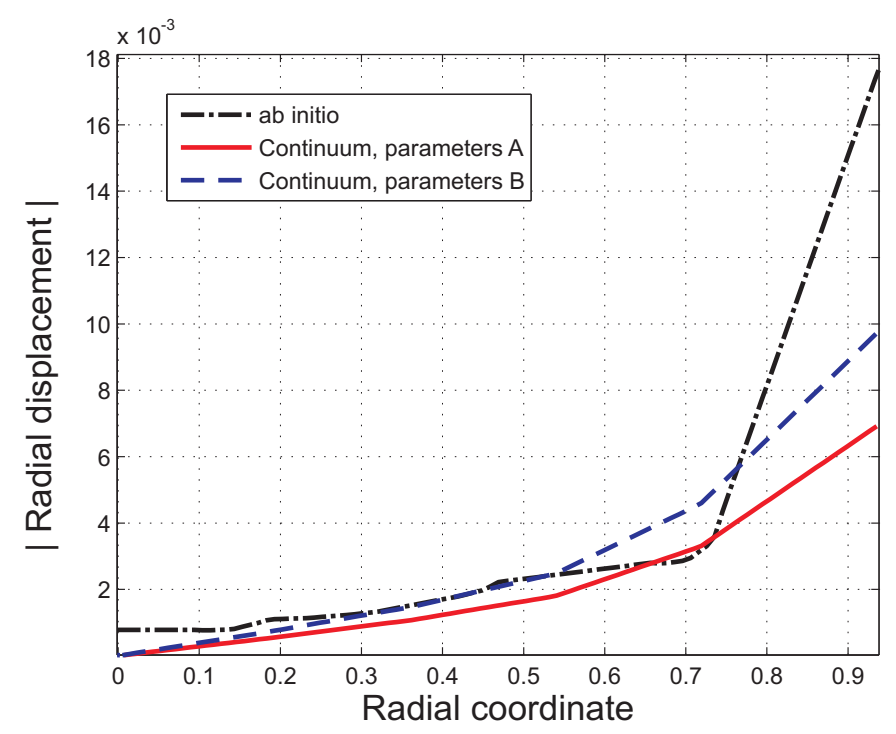

Fig. 11. Radial displacement field along nanowire radial direction $(\mathrm{d}=1.87 \mathrm{~nm})$. Comparison between continuum model and ab initio interpolated atomic displacements.

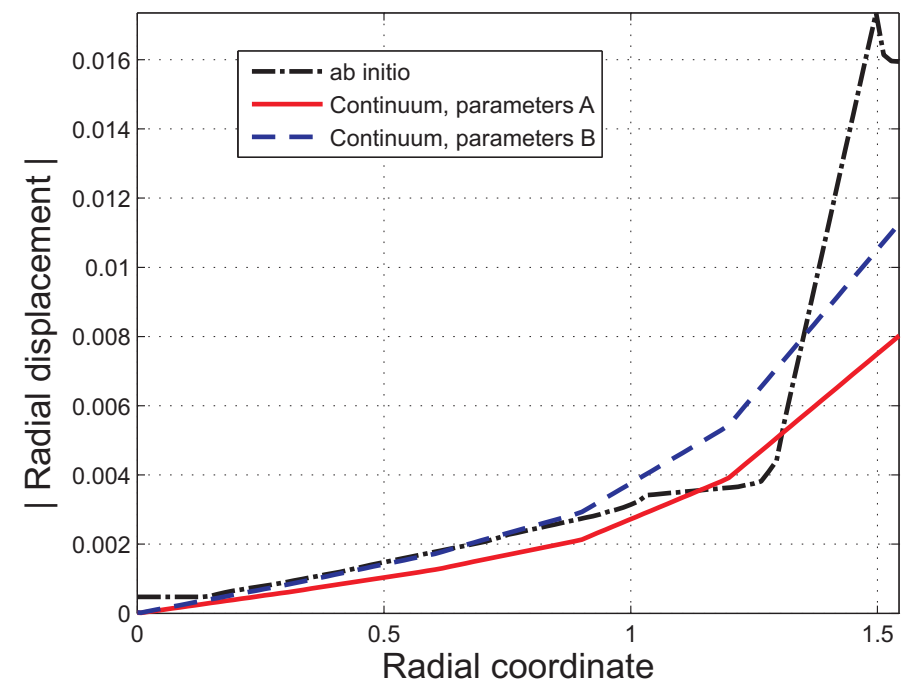

Fig. 12. Radial displacement field along nanowire radial direction $(\mathrm{d}=3.11 \mathrm{~nm})$. Comparison between continuum model and ab initio interpolated atomic displacements.

have very different kinematics, as shown in figure 13. On the surface layer, $\mathrm{Al}$ atoms possess a relatively large inward radial displacement as compared to the $\mathrm{Al}$ atoms in the next interior layer and with opposite sign to the $\mathrm{N}$ atoms which have an outward displacement on the surface. This highly heterogeneous field cannot be captured accurately by the proposed continuum model.

In figure 14 the radial strain field is depicted in nanowires with radii $2 \mathrm{~nm}, 5$ 

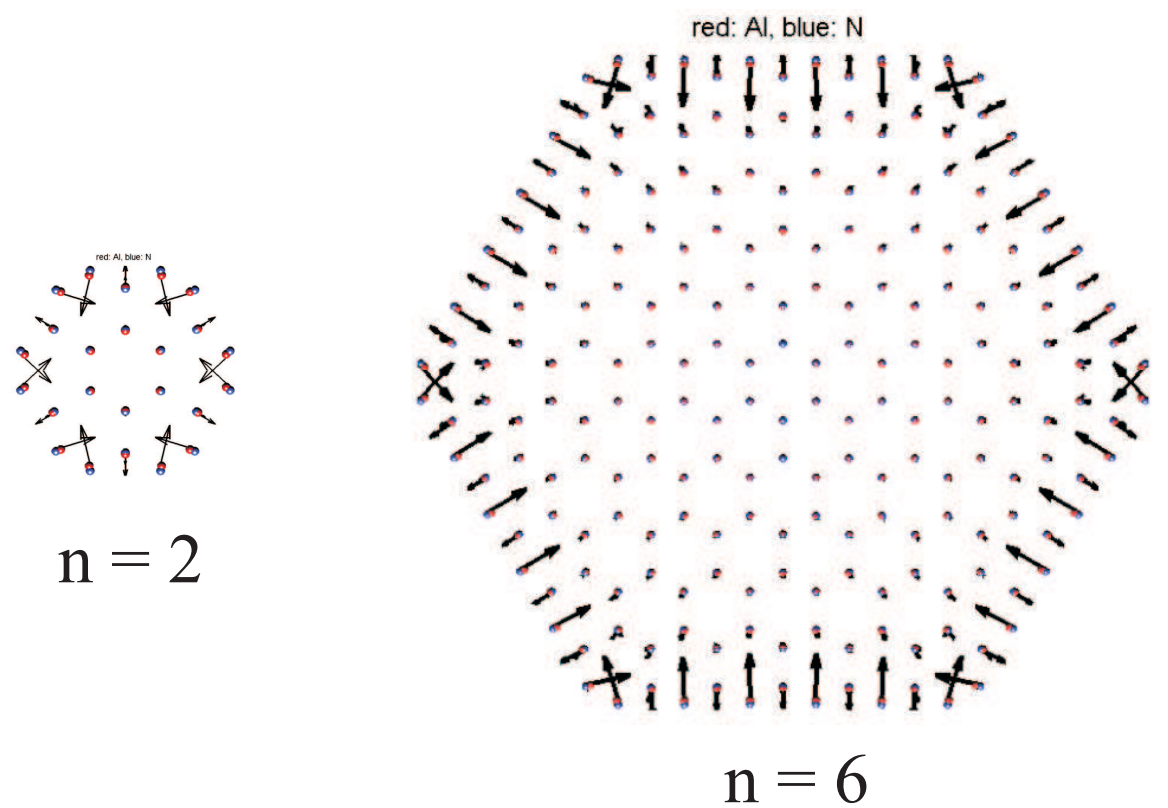

Fig. 13. Displacements in the nanowires in relaxation, ab initio calculations.

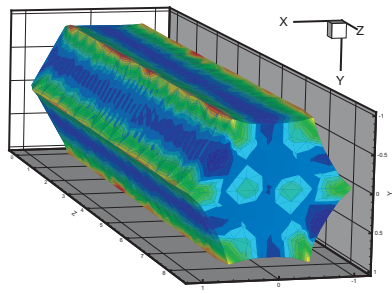

(a)

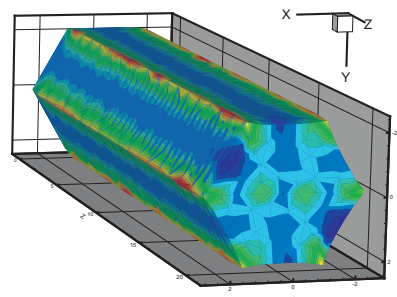

(b)

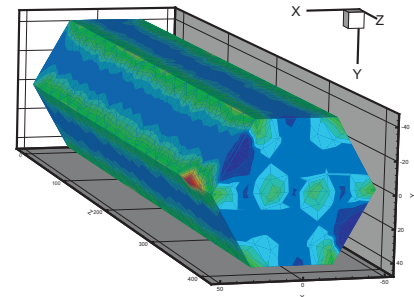

(c)

Fig. 14. Deformed configuration and radial strain fields in the nanowire for free relaxation (magnified 10 times); (a) d = $1 \mathrm{~nm}$; (b) d = $3 \mathrm{~nm}$; (c) d = $100 \mathrm{~nm}$.

$\mathrm{nm}$, and $100 \mathrm{~nm}$ in free relaxation, showing the influence of the size effects on the local strain distribution and amplitudes. The set of surface coefficients B was used.

In figure 15, configurations of the nanowire in free relaxation are presented, demonstrating the size effects on the deformed configuration.

\subsection{Bending of a nanowire}

In this example, the constructed continuum model is employed to study size effects in bending analysis of a nanowire. This problem is not tractable via $a b$ initio calculations, as no periodicity can be considered. The boundary conditions are depicted schematically in figure 16. This test is of practical importance, as this type of loading can be prescribed for example using an atomic 


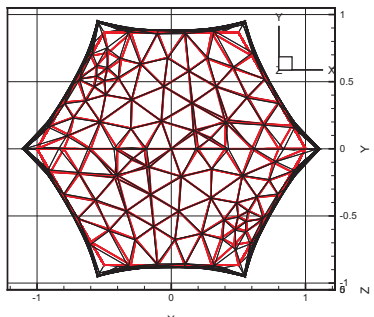

(a)

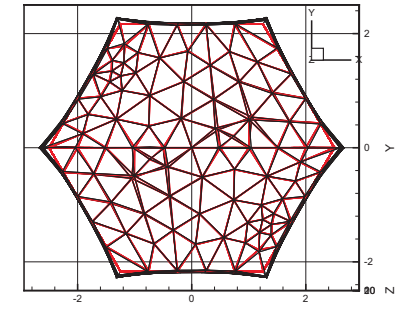

(b)

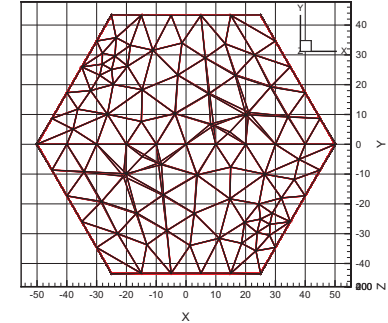

(c)

Fig. 15. Deformed configuration of the nanowire in free relaxation (magnified 10 times) for (a) $d=2 \mathrm{~nm}$; (b) $d=5 \mathrm{~nm}$; (c) $d=100 \mathrm{~nm}$. Red and black meshes correspond to undeformed and deformed configuration, respectively.

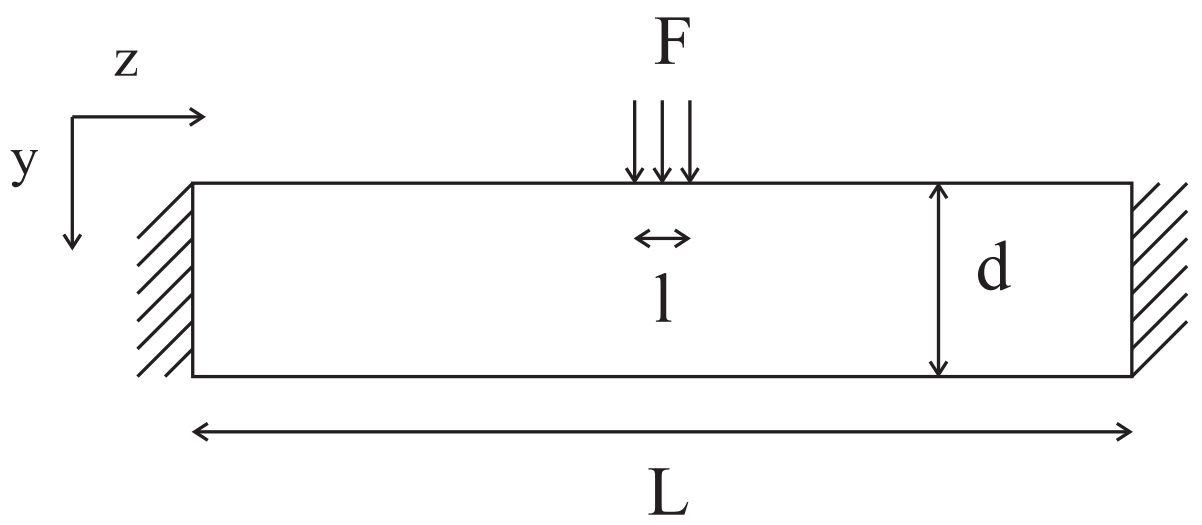

Fig. 16. Schematic view of the bending problem.

force microscope (AFM) [51]. The nanowire is clamped at its both ends and subjected to a force distribution $\mathbf{F}=\sigma_{y} \mathbf{e}_{y} \otimes \mathbf{e}_{y} \mathbf{n}, \sigma_{y}=1 e-8 \mathrm{MPa}$ on a width $l=L / 20$, as depicted in figure 16. As in previous example, the length of the nanowire is taken as $L=4 d$.

To evaluate the influence of surface effects, we compute the transverse stiffness defined as:

$$
K_{t}^{s}=\frac{F}{v}=\frac{\sigma_{y} d}{5 v}
$$

where $v$ is the deflection of the beam. The values are normalized with respect to the transverse stiffness of the beam $K_{t}^{b}$ when the surface effects are neglected. The results are presented in figure 17 . The size effects can be clearly observed. It is noteworthy that the choice of the surface parameters set does not significantly modify the results. As a conclusion, the coefficients extracted directly from the proposed slab procedure can be used without any further data fitting to provide a good approximation of the nanowire response when complex loading is prescribed.

In figures 18 and 19 the $\varepsilon_{y y}$ strain component and deformed configuration of the nanowire is depicted. Figure 19 shows the impact of the surface energy on 


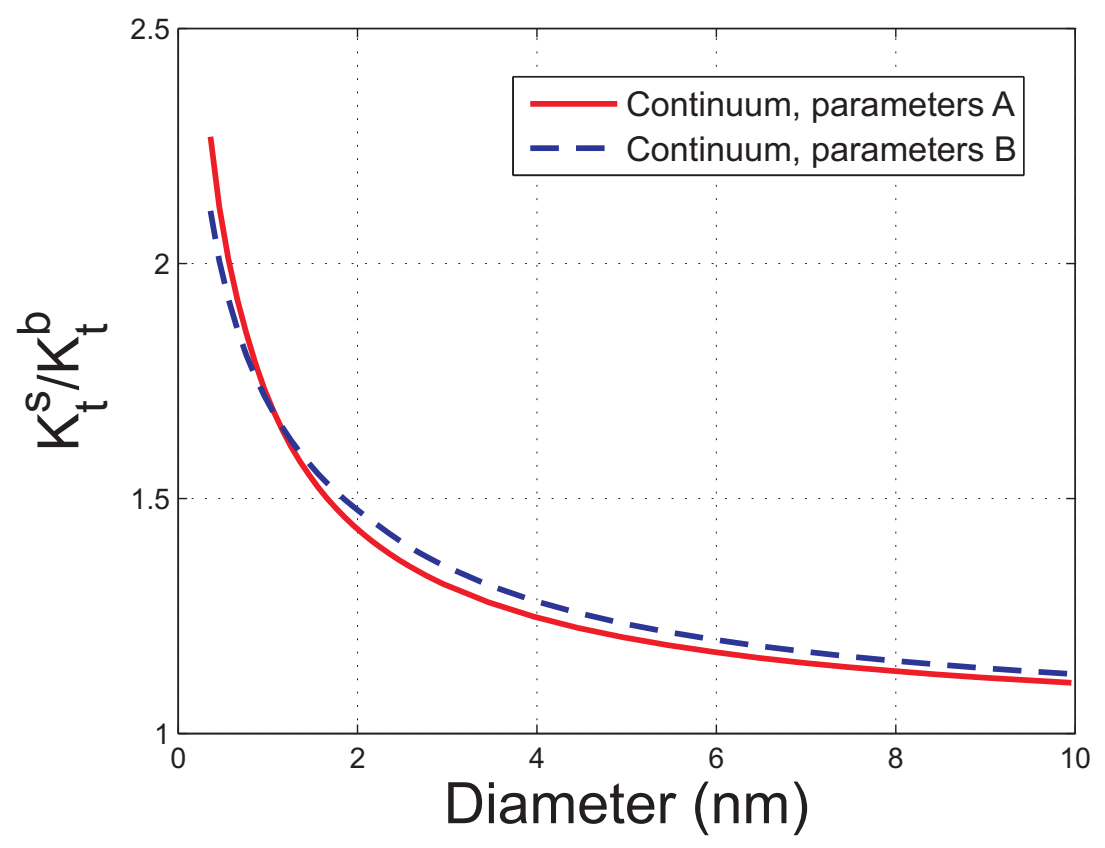

Fig. 17. Transverse stiffness of the nanowire versus diameter.

the local strain distribution for small nanowires.

\section{Conclusion}

A computational modeling framework has been proposed to simulate the response of nanowires including free surface effects. The additional surface energy due to the unbalanced atoms located near the surface are introduced in the continuum equations using the Gurtin-Murdoch elasticity theory and an appropriate weak form. A finite Element discretization procedure is described, where we avoid local basis coordinates in surface elements by employing projection operators. Finally, the study is completed by a methodology to extract via ab initio methods the elastic surface parameters. To validate our approach, a complete ab initio model of AlN nanowire is compared with the FEM model which uses the elastic properties extracted from surface models. Very good agreement is observed for different size-dependent properties such as Young modulus, or residual axial and radial strain. Finally, the model has been applied to study the size effects in bending analysis of a nanowire to illustrate the potential of the present approach for more complex loading cases, or when geometries and sizes are not reachable by atomistic simulations. Potential developments of this approach include extension to other materials and piezoelectrical effects. 


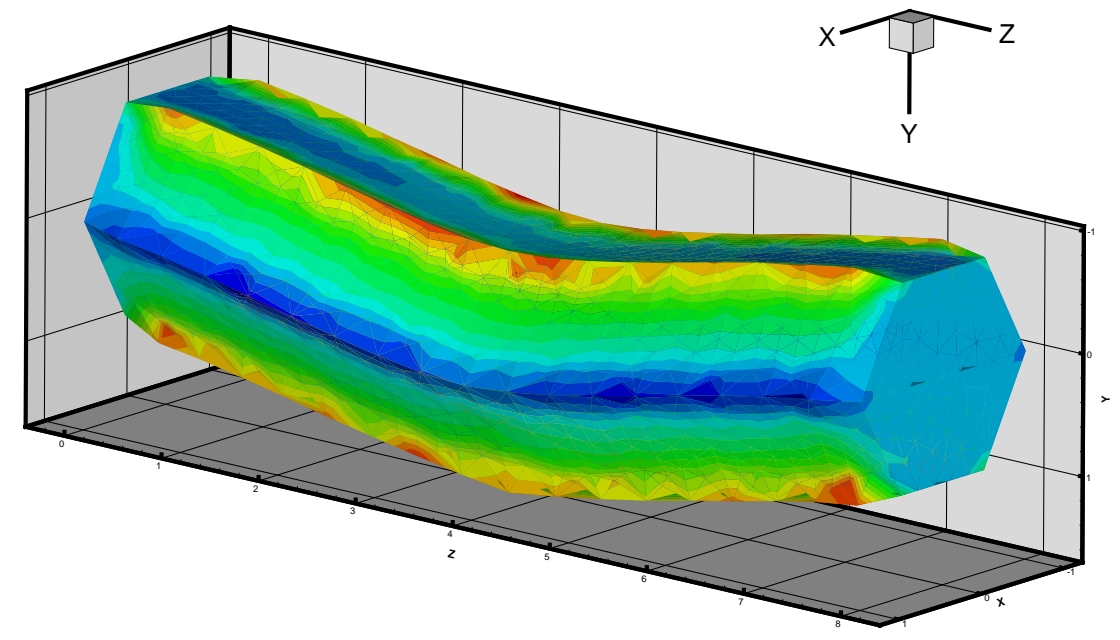

Fig. 18. $\varepsilon_{y y}$ strain field and deformed configuration of the nanowire in bending.
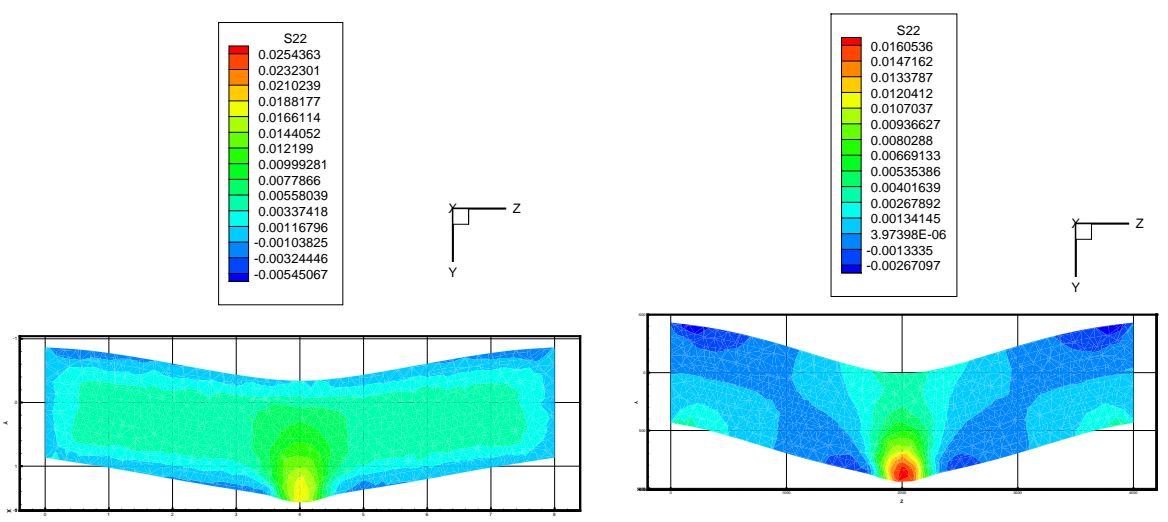

Fig. 19. $\varepsilon_{y y}$ strain field and deformed configuration of the nanowire in bending: size effects $(2 \mathrm{~nm}$ and $1000 \mathrm{~nm})$.

\section{Acknowledgments}

We are grateful to Dr. Shui-Tao Gu, Post-doctoral fellow at MSME, University Paris-Est, who carefully read our manuscript during the revision process and corrected typographic mistakes in the matrices expressions. 


\section{References}

[1] R. Agrawal, B. Peng, E.E. Gdoutos, H.D. Espinosa, Elasticity size effects in ZnO nanowires - A combined experimental - computational approach, Nano Lett. 8(11) (2008) 3668-3674.

[2] M.S. Arnold, P. Avouris, Z.W. Pan, Z.L. Wang, Field-effect transistors based on single semiconducting oxide nanobelts, J. Phys. Chem. B 107 (2003) 659-663.

[3] X.D. Bai, P.X. Gao, Z.L. Wang, E.G. Wang, Dual-mode mechanical resonnance of individual ZnO nanobelts, Appl. Phys. Lett. 82 (2003) 4806-4808.

[4] M. Born, On the stability of crystals, Proc. Cambridge Philos. Soc. 36 (1940) $160-172$.

[5] M. Cadek, J.N. Coleman, K.P. Ryan, V. Nicolosi, G. Bister, A. Fonseca, J.B. Nagy, K. Szostak, F. Beguin, W.J. Blau, Reinforcement of polymers with carbon nanotubes: the role of nanotube surface area, Nano Lett. 4 (2004) 353-356.

[6] M. Catti, Y. Noel, R. Dovesi, Full piezoelactric tensor of wurtzite and zinc blende $\mathrm{ZnO}$ and $\mathrm{ZnS}$ by first-principles calculations, J. of Phys. and Chem. of Solids. 64 (2003) 2183-2190.

[7] C.Q. Chen, Y. Shi, Y.S. Zhang, J. Zhu, Y.J. Yan, Size dependence of Young's modulus in ZnO nanowires, Phys. Rev. Lett. 96 (2006) 075505.

[8] T. Chen, M.-S. Chiu, C.-N. Weng, Derivation of the generalized Young-Laplace equation of curved interfaces in nanoscaled solids, J. Appl. Phys. 100 (2006) 074308 .

[9] W.-H. Chen, H.-C. Cheng, Y.-C. Hsu, R.-H. Uang, J.-S. Hsu, Mechanical material characterization of Co nanowires and their nanocomposites, Compos. Sci. Technol. 68 (2008) 2288-3395.

[10] Y. Cheng, P. Xiong, L. Fields, J.P. Zheng, R.S. Yang, Z.L. Wang, Intrinsic characteristics of semi conducting oxide nanobelts field-effect transistors, Appl. Phys. Lett. 89 (2006) 093114.

[11] E. Comini, G. Faglia, G. Sberveglieri, Z.W. Pan, Z.L. Wang, Stable and highly sensitive gas sensors based on semiconducting oxide nanobelts, Appl. Phys. Lett. 81 (2002) 1869-1871.

[12] H.G. Craighead, A Nano electromechanical systems, Science 290 (2000) 15321535 .

[13] S. Cuenot, C. Frétigny, S. Demoustier-Champagne, B. Nysten, Surface tension effect on the mechanical properties of nanomaterials measured by atomic force microscopy, Phys. Rev. B 69 (2004) 165410.

[14] L. Dai, W.C.D. Cheong, C.H. Sow, C.T. Lim, V.B.C. Tan, Molecular Dynamics Simulation of ZnO Nanowires: Size Effects, Defects, and Super Ductility, Langmuir 26(2) (2010) 1165-1171. 
[15] R. Dingreville, J. Qu, M. Cherkaoui, Surface free energy and its effects on the elastic behavior of nano-sized particles, wires and film, J. Mech. Phys. Solids 53 (2005) 1827-1854.

[16] R. Dovesi, V.R. Saunders, C. Roetti, R. Orlando, C. M. Zicovich-Wilson, F. Pascale, B. Civalleri, K. Doll, N.M. Harrison, I.J. Bush, Ph. D'Arco, M. Llunell, CRYSTAL06 User's Manual, University of Torino, Torino, 2006

[17] H.L. Duan, J. Wang, Z.P. Huang, B.L. Karihaloo, Size-dependent effective elastic constants of solides containing nano-inhomogeneities with interface stress, J. Mech. Phys. Solids 53(7) (2005) 1574-1596.

[18] H.L. Duan, J. Wang, Z.P. Huang, B.L. Karihaloo, Eshelby formalism for nanoinhomogeneities, Proc. R. Soc. A 461 (2005) 3335-3353.

[19] H.L. Duan, X. Yi, Z.P. Huang, J. Wang, A unified scheme for prediction of effective moduli of multiphase composites with interface effects. Part I: Theoretical framework, Mech. Mater. 39 (2007) 81-95.

[20] H. Edelsbrunner, E. Mücke, Three-dimensional alpha-shapes, ACM Trans. Graph. 13 (1994) 43-72.

[21] K.L. Ekinci, M.L. Roukes, Nanoelectromechanical systems, Rev. Sci. Instrum. 76 (2005) 061101

[22] P.J. Feibelman, Pulay-type formula for surface stress in a local-densityfunctional, linear combination of atomic orbitals, electronic-structure calculation, Phys. Rev. B 44 (1991) 3916-3925.

[23] J. Fish, W. Chen, Discrete-to-continuum bridging based on multigrid principles, Comput. Meth. Appl. Mech. Engrg. 193 (2004) 1693-1711.

[24] C. Gatti, V.R. Saunders,C. Roetti, Crystal-field effects on the topological properties of the electron-density in molecular-crystals - the case of urea, J. Chem. Phys. 101 (1994) 10686-10696.

[25] S.-T. Gu, Contributions to the modelling of imperfect interfaces and to the homogenization of heterogeneous materials, Ph.D thesis in French, Université Paris-Est, France (2008).

[26] M.E. Gurtin, A.I. Murdoch, Continuum theory of elastic-material surfaces, Arch. Ration. Mech. Anal. 57(4) (1975) 291-323.

[27] J.C. Hamilton, W.G. Wolfer, Theories of surface elasticity for nanoscale objects, Surf. Sci. 603 (2009) 1284-1291.

[28] Y. Huang, X. Bai, Y. Zhang, In situ mechanical properties of individual ZnO nanowires and the mass measurement of nanoparticles, J. Phys.: Condens. Matter 18 (2006) L179-L184.

[29] W.L. Hughes, Z.L. Wang, Nanobelts as nanocantilevers, Appl. Phys. Lett. 82 (2003) 2886-2888. 
[30] H. Iwanaga, A. Kunishige, S. Takeuchi, Anisotripic thermal expansion in wurtzite-type crystals, Phys. Rev. B 24 (1981) 5634.

[31] I.N. Levine, Quantum chemistry (2000), Prentice-Hall, New Jersey.

[32] A. Javili, P. Steinmann, A finite element framework for continua with boudary energies. Part I: The two-dimensional case, Comput. Methods Appl. Mech. Engrg. 198 (2009) 2198-2208.

[33] A. Javili, P. Steinmann, A finite element framework for continua with boudary energies. Part II: The three-dimensional case, Comput. Methods Appl. Mech. Engrg. 199 (2010) 755-765.

[34] H. Le Quang, Q.-C. He, Size-dependent effective thermoelastic properties of nanocomposites with spherically anisotropic phases, J. Mech. Phys. Solids, 55(9) (2007) 1899-1931.

[35] X.-F. Li, B.-L. Wang, K.Y. Lee, Size effects of the bending stiffness of nanowires, J. Appl. Phys. 105 (2009) 074306.

[36] C.M. Lieber, One-dimensional nanostructures: chemistry, physics and applications, Solid State Commun. 107 (1998) 607-616.

[37] R. Maranganti, P. Sharma, Atomistic determination of exoelectric properties of crystalline dielectrics, Phys. rev. B 80 (2009) 054109.

[38] R.E. Miller, V.B. Shenoy, Size-dependent elastic properties of nanosized structural elements, Nanotechnology 11 (2000) 139-147.

[39] A. Mitrushchenkov, G. Chambaud, J. Yvonnet, Q.-C. He, Towards an elastic model of wurtzite AlN nanowires, submitted.

[40] B. Montanari, B. Civalleri, C.M. Zicovich-Wilson, R. Dovesi, Influence of the exchange-correlation functional in all-electron calculations of the vibrational frequencies of corundum (alpha-Al2O3), Int. J. Quantum Chem. 106 (2006) 17031714 .

[41] H.S. Park, P.A. Klein, G.J. Wagner, A Surface Cauchy-Born model for nanoscale materials, Int. J. Numer. Meth. Engrg. 68 (2006) 1072-1095.

[42] H.S. Park, P.A. Klein, Surface Cauchy-Born analysis of surface stress effects on metallic nanowires, Phys. Rev. B 75 (2007) 085408.

[43] H.S. Park, Strain sensing through the resonnant properties of deformed metal nanowires, J. Appl. Phys. 104 (2008) 013516.

[44] H.S. Park, P.A. Klein, A surface Cauchy-Born model for silicon nanostructures, Comput. Methods Appl. Mech. Engrg. 197 (2008) 3249-3260.

[45] H.S. Park, W. Cai, H.D. Espinosa, H. Huang, Mechanics of crystalline nanowires, MRS Bull. 34 (2009) 178-183.

[46] J. Qi, D. Shi, J. Jia, First-principles studies of the electronic and mechanical properties of $\mathrm{ZnO}$ nanobelts with different dominant surfaces, Nanotechnology 19 (2008) 435707. 
[47] C.D. Reddy, A. Ramasuramaniam, VB Shenoy, Y.-W. Zhang, Edge elastic properties of defect-free single-layer graphene sheets, Appl. Phys. Lett. 94 (2009) 101904.

[48] R.E. Rudd, J.Q. Broughton, Coarse-grained molecular dynamics and the atomic limit of finite elements, Phys. Rev. B 58 (1998) 5893-5896.

[49] B.A. Samuel, A.V. Desai, M.A. Haque, Design and modeling of a MEMS picoNewton loading/sensing device, Sens. Actuators A 127(1) (2006) 155-162.

[50] A.R. Sethoodeh, H. Attariani, M. Khosrownejad, Nickel nanowires under uniaxial loads: a molecular dynamics simulation study, Comput. Meterials. Science 44 (2008) 378-384.

[51] S. Shanmugham, J. Jeong, A. Alkhateeb, D.E. Aston, Polymer nanowire elastic moduli measured with digital pulsed force mode AFM, Langmuir 21 (2005) 10214-10218.

[52] P. Sharma, S. Ganti, N. Bhate, Effects of surface on the size-dependent elastic state of nano-inhomogeneities, Appl. Phys. Lett. 82 (2003) 535-537.

[53] P. Sharma, S. Ganti, Size-dependent Eshelby's tensor for embedded nanoinclusions incorporating surface/interface energies, ASME J. Appl. Mech. 71 (2004) 663-671.

[54] V.B. Shenoy, Atomistic calculations of elastic properties of metallic fcc crystal surfaces, Phys. Rev. B 71 (2005) 094104.

[55] R.P. Singh, M. Zhang, D. Chan, Toughening of a brittle thermosetting polymer: effects of reinforcement particle size and volume fraction, J. Mater. Sci. 37 (2002) 781-788.

[56] J. Song, X. Wang, E. Riedo, Z.L, Wang, Elastic properties of vertically aligned nanowires, Nano Lett. 5(10) (2005) 1954-1958.

[57] I. Stakgold, The Cauchy relations in a molecular theory of elasticity, Quart. Appl. Mech. 8 (1950) 169-186.

[58] E. Tadmor, M. Ortiz, R. Phillips, Quasicontinuum analysis of defects in solids, Philos. Mag. A 73 (1996) 1529-1563.

[59] L. Tian, R.K.N.D. Rajapakse, Finite element modelling of nanoscale inhomogeneities in an elastic matrix, Comput. Mater. Sci 41 (2007) 44-53.

[60] G.J. Wagner, W.K. Liu, Coupling of atomistic and continuum simulations using a bridging scale decomposition, J. Comput. Phys. 190 (2003) 249-274.

[61] X. Wang, J. Song, J. Liu, Z. L. Wang, Coupling of atomistic and continuum, Science 316 (2007) 102.

[62] J. Wang, H.L. Duan, Z.P. Huang, B.L. Karihaloo, A scaling law for properties of nano-structured materials, Proc. R. Soc. Lond. A 462 (2006) 1355-1363. 
[63] G. Wang, X. Li, Predicting Young's modulus of nanowires from first-principles calculations on their surface and bulk materials, J. Appl. Physics 104 (2008) 113517 .

[64] G. Wei, Y. Shouwen, H. Ganyun, Finite element characterization of the sizedependent mechanical behaviour in nanosystems, Nanotechnology 17 (2006) 1118-1122.

[65] J.H. Weiner, Hellman-Feynman theorem, elastic moduli, and the Cauchy relation, Phys. Rev. B 24 (1981) 845-848.

[66] D. Whang, S. Jim, Y. Wu, C.H. Lieber, Large-scale hierarchical organization of nanowire arrays for integrated nanosystems, Nano lett. 3(9) (2003) 1255-1259.

[67] E.W. Wong, P.E. Sheehan, C.M. Lieber, Nanobeam mechanics: Elasticity, strength, and toughening of nanorods and nanotubes, Science 277 (1997) 19711975 .

[68] H.A. Wu, X.X. Wang, An atomistic-continuum inhomogeneous material model for the elastic bending of metal nanocantilevers, Adv. Eng. Softw. 39 (2008) 764-769.

[69] Y. Xi, C.G. Hu, X.Y. Han, Y.F. Xiong, P.X. Gao, G.B. Liu, Hydrothermal synthesis of $\mathrm{ZnO}$ nanobelts and gas sensitivity property, Solid State Commun. 141 (2007) 506-509.

[70] S.P. Xiao, T. Belytschko, A bridging domani method for coupling continua with molecular dynamics, Comput. Meth. Appl. Mech. Engrg. 193 (2004) 1654-1669.

[71] J. Yvonnet., H. Le Quang, Q.-C. He, An XFEM/level set approach to modelling surface/interface effects and to computing the size-dependent effective properties of nanocomposites, Comput. Mech. 42 (2008) 704-712.

[72] J. Zhang, T. Wu, W. Jiang, L. Chen, Microstructure and properties of $\mathrm{Ti}_{3} \mathrm{SiC}_{2} / \mathrm{SiC}$ nanocomposites fabricated by spark plasma sintering, Compos. Sci. Technol. 68 (2008) 499-505.

[73] T.-Y. Zhang, M. Luo, W.K. Chan, Size-dependent surface stress, surface stiffness, and Young's modulus of hexagonal prism [111] $\beta$-SiC nanowires, J. Appl. Phys. 103 (2008) 104308.

[74] Y. Zhang, J. Hong, B. Liu, D. Fang, Molecular dynamics investigations on the size-dependent ferroelectric behavior of BaTiO3 nanowires, Nanotechnology 20 (2009) 405703.

[75] R. Zhu., D. Wang, S. Xiang, Z. Zhou, X. Ye, Zinc oxide nanowire electromechanical oscillator, Sens. Actuator A-Phys. 154 (2009) 3249-3260. 